\title{
Disability Training for Health Workers: A Global Narrative Systematic Review
}

Sara Rotenberg, BS ; ; Danae Rodríguez Gatta, BSc., MSc. ${ }^{\text {; }}$ Azizia Wahedi, HBSc., MSc. ${ }^{\text {; }}$, Rachelle Loo, BSc. ${ }^{\text {; }}$ Emily McFadden, MA, MSc, PhD, ${ }^{\text {e; }}$ Sara Ryan, BSc, PhD, ${ }^{f}$

${ }^{a}$ Nuffield Department of Primary Care Health Sciences, University of Oxford

${ }^{\mathrm{b}}$ International Centre for Evidence in Disability, London School of Hygiene and Tropical Medicine

${ }^{\mathrm{c}}$ Medical Sciences Division, University of Oxford

${ }^{\mathrm{d}}$ Cumming School of Medicine, University of Calgary

${ }^{\mathrm{e}}$ Centre for Evidence-Based Medicine, University of Oxford

${ }^{\mathrm{f}}$ Faculty of Health, Psychology, and Social Care, Manchester Metropolitan University

\section{ABSTRACT}

Background: Health worker training on disability is a recognized component of achieving high standards of health for people with disabilities, given that health worker's lack of knowledge, stigma, and negative attitudes towards people with disabilities act as barriers to high quality health care.

Objective: To understand the published literature on training health workers about disability.

Methods: We searched five databases for relevant peer-reviewed articles published between January 2012 and January 2021. Studies that focused on training health care workers to improve knowledge, confidence, self-efficacy, and competence to support people with physical, sensory, or intellectual impairments were included. Data about the details of the intervention (setting, participants, format, impact assessments, etc.) and its effects were extracted.

Results: There is an array of highly local tools to train health workers across stages of their training and careers (pre-service, in-service, and continuing professional development). Studies involving people with disabilities in the training, community placements, simulations, or interactive sessions were found to be most effective in improving knowledge, confidence, competency, and self-efficacy.

Conclusions: As part of initiatives to build inclusive health systems and improve health outcomes for people with disabilities, health workers around the world need to receive appropriate and evidence-based training that combine multiple methods and involve people with disabilities. 
medRxiv preprint doi: https://doi.org/10.1101/2021.08.03.21261522; this version posted August 4, 2021. The copyright holder for this preprint (which was not certified by peer review) is the author/funder, who has granted medRxiv a license to display the preprint in perpetuity.

It is made available under a CC-BY-ND 4.0 International license .

\section{BACKGROUND}

Human resources for health are at the heart of high-quality health systems. It is critical to improve health worker training to improve health care for populations that are systematically marginalized by health systems, such as people with disabilities. The World Health Organization (WHO) estimates that people with disabilities make up 15\% of the world's population. ${ }^{1}$ People with disabilities often face significant barriers to health care, including lack of accessible transport and facilities, limited financial protection, poor health worker attitudes that result in worse outcomes or limited health worker training on disability. ${ }^{2}$ Even in countries where there is guaranteed universal access and financial protection, health workers' unfamiliarity with disability, or negative attitudes towards people with disabilities, can not only foster an unwelcoming environment, but also contribute to high rates of patient safety issues and poor quality care. ${ }^{1}$

Health worker training on disability is a recognized component of achieving high standards of health for people with disabilities. While the UN Convention on the Rights of Persons with Disabilities (UNCRPD) Article $25^{3}$ has specific requirements on access to health care and SDG monitoring on health worker disability training, the recent World Health Assembly resolution most eminently highlights the role of health worker training in removing barriers to health care for people with disabilities. ${ }^{4}$ In addition, recent studies have highlighted the need to improve health care workers' attitudes, knowledge, and competency to provide care for people with disabilities. For example, a US study illustrated that just $40.7 \%$ of physicians were confident about providing care to patients with disabilities and most (82.4\%) perceived that people with significant disabilities have worse quality of life. Similarly, a study found that $87 \%$ of nursing 
students implicitly associated negative traits with physical disability, which may influence clinician behaviour. These studies illustrate the need to improve health workers' confidence, competency, attitudes, and comfort in treating patients with disabilities. Given these international agreements and recent studies, it is important that countries around the world begin to integrate disability training systematically and use examples of successful interventions as models.

This review directly builds on a previous review by Shakespeare and Kleine that explored health worker training on disability between 2000 and $2011 .^{5}$ The study found that, while there are numerous interventions to teach medical professionals about disability, there are few common philosophical underpinnings, insufficient hands-on experience, and more opportunities to incorporate disability across the curriculum. ${ }^{5}$ Since this review, additional systematic reviews have examined health worker training on people with disabilities for certain populations of health workers ${ }^{6}$, certain impairments, ${ }^{7}$ or geographic areas.

Given renewed international commitments to health worker training on disability and countrylevel plans in Australia ${ }^{8}$ and the $\mathrm{UK}^{9}$ to train health workers on specific types of disability, it is important to update Shakespeare and Kleine's review ${ }^{5}$ and outline the types of interventions to improve health worker's knowledge, confidence, self-efficacy, and competence in treating patients with disabilities. Understanding the ways in which health workers receive training on disability, will facilitate adjusting system-level policies and individual-level practices to improve care for people with disabilities. Ultimately, this review will help to understand the types of training that support positive and sustained improvements in service delivery for health workers serving people with disabilities. 


\section{METHODS}

\section{Search Strategy}

Electronic searches were conducted for the EMBASE, Global Health, Medline, CINAHL, ASSIA and Web of Science databases between 18-19 January 2021. Search terms were developed in three domains: disability, health education, and health workers. Disability terms were general, focusing on various types of impairments; health education terms targeted aspects of health training (i.e., 'core competency', 'patient encounter', 'standardized patient', etc.); and health worker terms were developed using key terms from WHO's International Classifications of Health Workers. ${ }^{10}$ Terms were developed using MeSH, keywords, or equivalent as well as from other reviews on similar topics and searches were limited to papers in English, French, or Spanish. These parameters and strategy were agreed upon by the authors and a research librarian before the search was conducted to ensure there were adequate words to capture articles across the three domains examined. The Preferred Reporting Items for Systematic Reviews and MetaAnalysis (PRISMA) statement was followed for conducting and reporting the review (PROSPERO Registration: CRD42021231120). All studies identified by the review were exported into an EndNote database (version X20, Clarivate Analytics, Philadelphia, PA, USA) and then exported into Rayyan (Qatar Computing Research Institute, Qatar) for screening. ${ }^{11}$

\section{Selection Criteria}

Our search strategy sought to identify peer-reviewed articles from around the world published between January 2012 and 2021. Given a previous systematic review covered this topic until $2011^{5}$, the search included articles published from 2012-January 2021 and included all health worker types, health education levels, and disability globally. The inclusion criteria required that 
studies were: qualitative and/or quantitative in methods; included a complete description of the intervention; explicit evaluation of the training's impact (i.e., pre- and/or post-training evaluations, follow-up surveys, etc.); and had a particular focus on improving disability competency, knowledge, confidence, self-efficacy, curricula, or teaching methods. Studies examining health worker attitudes towards people with disabilities were excluded on the basis that a positive attitude does not necessarily guarantee improved competency or care outcomes. Studies that measured attitudes alongside other criteria were included. Finally, the abundance of articles on training health workers about mental health, the authors decided that this topic merited further, independent exploration, and, therefore, we excluded papers that trained health workers only about mental health. Only papers that looked at physical, sensory, intellectual or developmental impairments were included.

\section{Data Extraction}

All data were extracted into a Google Sheet developed for this review. 78 full-text articles underwent data extraction, following a title, abstract, and full-text review by two reviewers (SRo and DR). An additional three reviewers (SRo, AW, RL) extracted data related to the general study information, setting, country, health worker cadre, number of participants, type of disability, features of the intervention, impact measurement, and outcomes. The extraction was double-checked by a second reviewer and collectively checked again by the extractors (SRo, AW, RL). Any conflicts in inclusion or extraction were resolved through discussion with a third and/or fourth member of the review team.

Fig 1. Flow chart of selected studies to review health worker training on disability ${ }^{12}$ 
medRxiv preprint doi: https://doi.org/10.1101/2021.08.03.21261522; this version posted August 4, 2021. The copyright holder for this preprint (which was not certified by peer review) is the author/funder, who has granted medRxiv a license to display the preprint in perpetuity.

It is made available under a CC-BY-ND 4.0 International license .

\section{Identification of studies via databases}

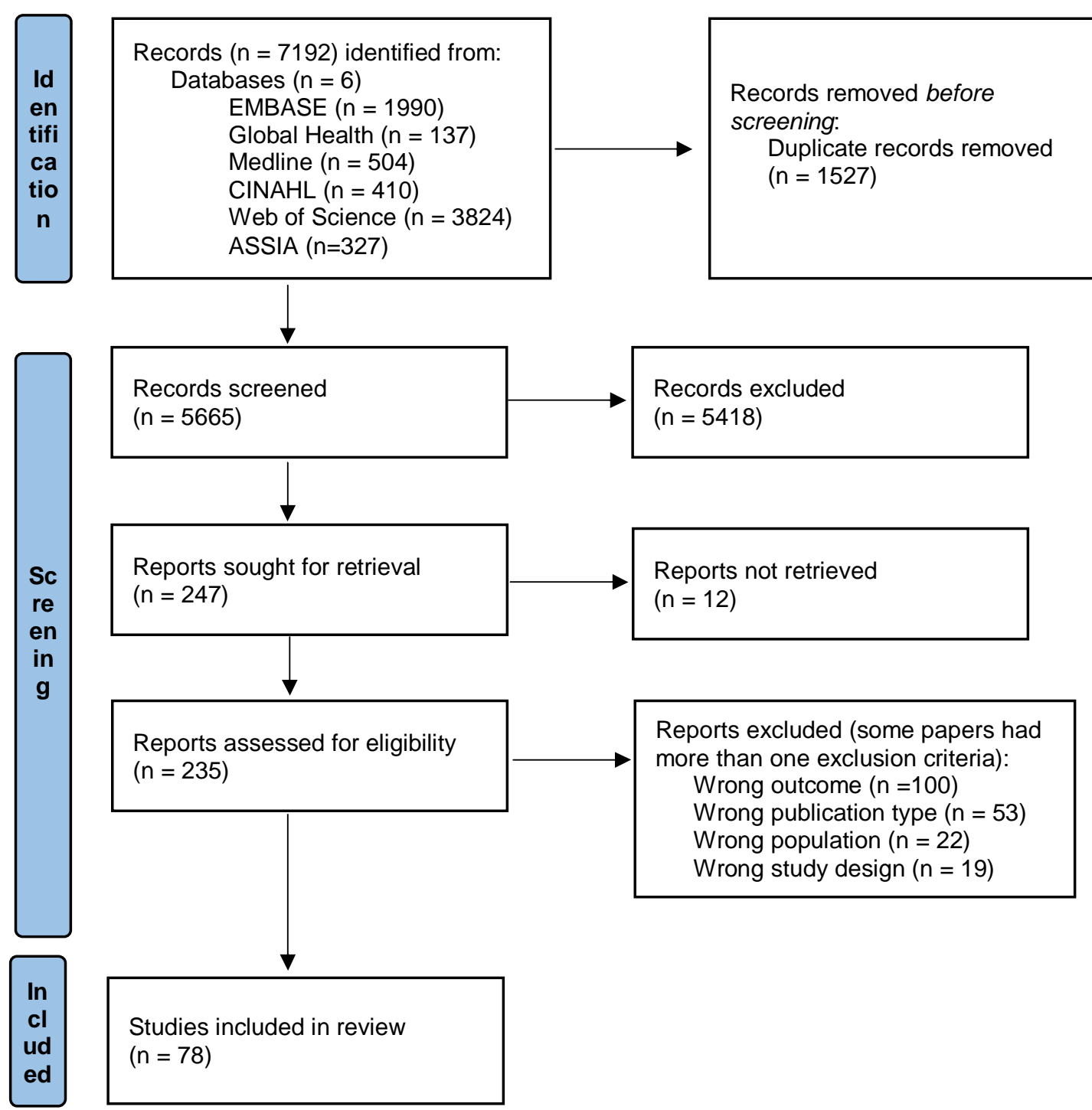

Given the wide array of study instruments and outcomes used to assess training impact, a metaanalysis could not be conducted, and a narrative synthesis was conducted instead. Quality and bias assessments were conducted by at least two reviewers in accordance with the SIGN50 (Scottish Intercollegiate Guidelines Network Checklists) and modified slightly from previous methods. $^{13}$ 
medRxiv preprint doi: https://doi.org/10.1101/2021.08.03.21261522; this version posted August 4, 2021. The copyright holder for this preprint (which was not certified by peer review) is the author/funder, who has granted medRxiv a license to display the preprint in perpetuity.

It is made available under a CC-BY-ND 4.0 International license .

Studies were rated as low bias, if all or almost all of the criteria were fulfilled, and those that were not fulfilled were thought unlikely to alter the conclusions of the study; medium, if some of the criteria were fulfilled, and those not fulfilled were thought unlikely to alter the conclusions of the study; or high, if few or no criteria were fulfilled, and the conclusions of the study were thought likely or very likely to alter with their inclusion.

\section{RESULTS}

The preliminary search identified 5,665 articles for title and abstract screening, after 1,527 duplicates were removed. Following screening, 247 articles were included for full-text review. Twelve studies were excluded because full-texts could not be retrieved and a further 154 studies did not meet the inclusion criteria as shown in Fig.1., and 3 articles included in the review were excluded during extraction because of an unclear intervention $(n=1)$ and wrong population group $(n=2) .{ }^{14}$

Included studies $(n=78)$ represented a range of geographies, health workers, and intervention types. Among these, there were studies from 19 countries, including seven low- and middleincome countries. ${ }^{15}$ Most studies took place in the United States $(n=35)$, followed by the United Kingdom ( $\mathrm{n}=13)$. Among the studies included, 30 were rated as low, 43 as medium, and five as high risk of bias [Supplementary Materials]. Studies varied in whether they were mandatory or optional; free or paid; and for certification or elective; however, many studies did not include this information. Various cadres of health workers were included in the study; doctors, medical students or residents $(n=37)$, and nurses or nursing students $(n=17)$ were the main recipients of training. These health workers were generally trained in the pre-qualification stage $(n=52)$, though there were several in-service $(n=7)$ and continuing professional development (CPD) programs $(n=19)$. The review included studies across disability groups; the most common focus 
medRxiv preprint doi: https://doi.org/10.1101/2021.08.03.21261522; this version posted August 4, 2021. The copyright holder for this preprint (which was not certified by peer review) is the author/funder, who has granted medRxiv a license to display the preprint in perpetuity.

It is made available under a CC-BY-ND 4.0 International license .

was training about people with intellectual and developmental disabilities $(n=41)$, followed by general programs about people with disabilities $(n=16)$. Most studies measured improvements in knowledge $(n=57)$ and competence $(n=42)$ outcomes, yet most studies used a self-designed evaluation instrument $(n=54)$. There was a wide variety of techniques to train health workers about disability, including lectures or other didactic methods $(n=65)$, and case studies $(n=28)$; the majority of studies $(n=58)$ used multiple teaching modalities [Appendix A].

\section{Lecture/Didactic Methods}

Most ( $n=65)$ studies included lectures or didactic methods, such as videos, multi-media formats, or online coursework. Many studies used these opportunities to introduce health professionals to the topic of disability from a rights-based perspective to enhance attitudes, awareness, and knowledge about disability. Some studies also taught particular skills that could be applied inpractice, such as an elective sign language class for medical and pharmacology terms ${ }^{16}$ to improve skills for engaging with $d$ /Deaf or hard of hearing individuals. Lectures were often combined with some other intervention (case study or simulation) to apply knowledge learned from the lecture. Participants in combined programs identified the content to be quite engaging and contributed to greater improvements in key outcomes. However, for those who only completed lecture or didactic-based methods, there were still improvements in the general

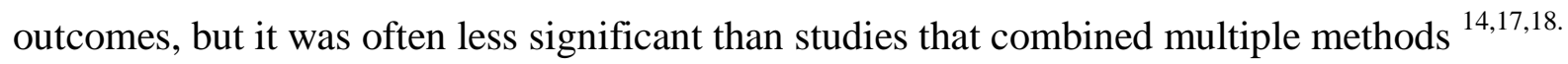
Finally, some programs utilized novel, innovative technology and multimedia tools to teach about disability in a more engaging method than traditional lectures or didactic methods. For example, one study designed multi-media tools (MMLTs) to teach medical students about common visual impairments and compared the knowledge scores with those who had read a 
medRxiv preprint doi: https://doi.org/10.1101/2021.08.03.21261522; this version posted August 4, 2021. The copyright holder for this preprint (which was not certified by peer review) is the author/funder, who has granted medRxiv a license to display the preprint in perpetuity.

It is made available under a CC-BY-ND 4.0 International license .

textbook. The findings highlight the importance of engaging material, as while there was no significant difference in knowledge (except for cataract recognition), the MMLT took less time and $87 \%$ of individuals found it more enjoyable than traditional teaching methods. ${ }^{19}$

\section{People with Disabilities as Teachers}

Recognizing the important role of self-advocates and patients as educators ${ }^{5}$, several studies $(n=19)$ invited people with disabilities to share their experiences in the health system, portray standardized patients, or give a lecture. Some universities hired people with disabilities to participate in simulated patient programs, while others asked patients to participate voluntarily. Many others found creative ways of engaging with people with disabilities. Cardiff University, for example, hired a self-advocacy theatre group to run a simulation and icebreaker activity. ${ }^{20}$ These activities added a non-clinical dimension to medical training about disability, as it allowed participants to explore disability outside the health worker-patient relationship and engage in dialogue. Studies that measured participants comfort and attitudes before and after a person with a disability as a teacher demonstrated that participants felt the non-clinical interaction enhanced their comfort and attitudes towards people with disabilities. ${ }^{21,22}$ 
Table 1: Characteristics of Included Studies

\begin{tabular}{|c|c|c|}
\hline Variable & Number & Percentage $(\%)$ \\
\hline \multicolumn{3}{|c|}{ Training Location } \\
\hline North America & 40 & 51.2 \\
\hline Europe & 22 & 28.2 \\
\hline Asia & 6 & 7.7 \\
\hline Oceania & 4 & 5.1 \\
\hline Africa & 4 & 5.1 \\
\hline South America & 2 & 2.5 \\
\hline \multicolumn{3}{|c|}{ Disability Focus of Training** } \\
\hline Intellectual and developmental & 41 & 51.9 \\
\hline All types of disabilities/unspecified & 16 & 20.3 \\
\hline Physical/motor & 13 & 15.2 \\
\hline Communication & 9 & 11.4 \\
\hline D/deaf and hard of hearing & 8 & 10.1 \\
\hline Visual impairment & 3 & 3.8 \\
\hline Sensory impairment & 2 & 2.5 \\
\hline \multicolumn{3}{|c|}{ Health Worker Type** } \\
\hline Medics & 37 & 35.2 \\
\hline Nurses & 17 & 16.2 \\
\hline Occupational and Physical Therapists & 17 & 15.2 \\
\hline Allied Health Professionals & 8 & 7.6 \\
\hline Dentists & 7 & 6.7 \\
\hline Audiologists and Speech Language Pathologists & 6 & 5.7 \\
\hline Psychologists & 4 & 3.8 \\
\hline Personal Care Workers & 5 & 4.8 \\
\hline Community Health Workers & 2 & 1.9 \\
\hline Pharmacists & 2 & 1.9 \\
\hline \multicolumn{3}{|c|}{ Teaching Method* } \\
\hline Lecture/didactic methods & 65 & 34.7 \\
\hline Case study & 28 & 14.9 \\
\hline Clinical encounter & 26 & 13.9 \\
\hline Placements, experiential, and community-based learning & 25 & 13.4 \\
\hline Simulation & 24 & 12.8 \\
\hline People with disabilities as a teacher & 19 & 10.1 \\
\hline \multicolumn{3}{|c|}{ Intervention Outcomes* } \\
\hline Knowledge & 57 & 28.4 \\
\hline Competence & 42 & 20.9 \\
\hline Attitudes & 31 & 15.4 \\
\hline Confidence & 24 & 11.9 \\
\hline Comfort & 15 & 7.5 \\
\hline Communication skills & 12 & 6.0 \\
\hline Self-Efficacy & 11 & 5.5 \\
\hline Other related outcomes & 9 & 4.5 \\
\hline
\end{tabular}


medRxiv preprint doi: https://doi.org/10.1101/2021.08.03.21261522; this version posted August 4, 2021. The copyright holder for this preprint (which was not certified by peer review) is the author/funder, who has granted medRxiv a license to display the preprint in perpetuity.

It is made available under a CC-BY-ND 4.0 International license .

\section{Case Studies}

Case studies are a common tool in medical education to prepare health workers holistically for their education, provided they have clear structure, details, and observations. ${ }^{23}$ Accordingly, many studies used case studies $(n=28)$ as a way of learning how to improve care for people with disabilities. These tools were especially common in continuing professional development, as some programs ask patients to bring case examples to review anonymously to improve care 24,25 or were to spur reflection on their own work. Several case studies were conducted through online learning or innovative interactive methods. For example, the City University of London created CitySCaPE, which is a multi-media simulation that simulated different patient cases of people with intellectual disabilities with nursing students. ${ }^{26}$ These types of case studies that blended the traditional case study and simulation aspects created greater engagement in settings where inperson or clinical encounters were not possible.

\section{Placements, experiential, and community-based learning}

Placements, experiential, and community-based learning $(n=25)$ methods were sustained opportunities to engage with people with disabilities in clinical and alternative settings that were common in in-service and pre-qualification training. For example, some studies examined the impact of clerkship placements in specialized clinics for people with disabilities, ${ }^{27}$ while others looked at nurses and occupational therapists' improvements after participating in a week-long summer camp for children with disabilities. ${ }^{28}$ In the clinical setting, students found that they improved skills because they were able to engage with people with disabilities for extended periods, rather than a singular interaction. Furthermore, the out-of-clinic engagement, such as at camps, schools, or residential settings helped illustrate the non-medical and everyday lives of people with disabilities. 
medRxiv preprint doi: https://doi.org/10.1101/2021.08.03.21261522; this version posted August 4, 2021. The copyright holder for this preprint (which was not certified by peer review) is the author/funder, who has granted medRxiv a license to display the preprint in perpetuity.

It is made available under a CC-BY-ND 4.0 International license .

\section{Simulations}

Simulations were found to be helpful tools to support skill development and learning outside of health worker-patient interactions. Many speech-language pathology, nursing, and medical student programs used simulations $(n=24)$ as tools to develop confidence and communication skills when treating patients with communication disorders. ${ }^{29,30,31}$ In addition, several medical schools integrated disability training into existing clinical simulation skills labs to improve care for people with disabilities. For example, at the University of Gothenburg medical students were videotaped during a simulated patient exercise to reflect on improving communications skills, particularly for the simulated patient with an acquired communication disorder. ${ }^{31}$ Overall, the simulations were useful tools for improving knowledge, comfort, and competency in a lowpressure environment that is applicable to serving people with disabilities.

\section{Clinical Encounters}

Several programs $(n=26)$ included singular clinical encounters with patients with disabilities as part of their disability training. These were often day-long programs to familiarize students with providing care and were predominantly focused on improving knowledge and competency. ${ }^{32}$ Most students who participated in these programs were advanced (i.e., penultimate or final year students) who had previously had some education on providing care to people with disabilities. These opportunities focused on practicing clinical skills to treat patients with disabilities, and, despite the short exposure, did significantly affect participant's key outcome scores. For instance, clinical encounters used in CPD, such as in Rwanda, where instructors in a physiotherapist training program went to participants' clinics to provide immediate feedback on their practice. ${ }^{33}$ 


\section{Multi-pronged approach}

Approximately $75 \%$ of papers utilized a combination of methods to have impact on training participants. These multi-pronged approaches helped reach various learning styles and cement learning. Two papers included in this review utilized all of the interventions measured in this paper. For example, two State University of New York medical colleges demonstrated the importance of integrating disability across health worker curricula, as all participants significantly improved their knowledge, attitudes, and core competencies in treating patients with disabilities. ${ }^{34}$ Similarly, the University of South Florida had a 12-week clinical clerkship that involved classroom simulations, lectures, case studies, people with disabilities as teachers, and a twice-weekly placement in a community clinic that served people with disabilities. The immersion helped to significantly improve knowledge, attitudes, and comfort. ${ }^{35}$

\section{DISCUSSION}

Numerous studies and examples serve as successful models to train health workers about disability and improve knowledge, competence, skills, self-efficacy, and confidence to treat patients with disabilities. All teaching methods had some positive impact on the outcomes measured in this study, regardless of health worker type, location, or training stage, though the most commonly used were lecture/didactic methods and case studies. Part of the success of these programs was the multi-pronged nature of the approach, as $75 \%$ of studies used multiple teaching methods. The two examples that utilized all teaching interventions demonstrate the importance of a multi-pronged approach that emphasizes mainstreaming disability in health curricula, either through sustained engagement in a curricula ${ }^{34}$ or an intensive placement. ${ }^{35}$ 
However, limited information about commonalities in curricula could be extracted from the data, given the diversity of interventions methods and topics.

It is important to note that these findings are not substantially different from the 2011 review. ${ }^{5}$ Similar methods are still used to teach health workers on disability and each example is highly localized, within either a certain school or region, other than two studies that examined nationallevel training programs. ${ }^{33,36}$ The limited evidence of systemic integration of disability training within health worker practices is concerning in this context as the current status of training appears to depend on where you received your training, where you live, or where you work. Enacting systemic-level change to ensure all health workers have the same level of high-quality training on disability will contribute to providing consistent, high-quality care and outcomes for people with disabilities.

Similarly, there was limited standardization in tools used to measure the impact of disability training on health workers. Of the 78 studies, nearly $70 \%$ designed their own instruments, and only two studies included the same standardized measure of outcomes. ${ }^{37,38}$ Few studies measured the longevity of the intervention's impact. Sustained approaches that mainstream disability should help to ensure learning is not performative for post-intervention evaluation, but actually effect change. This finding was previously noted ${ }^{5}$ and there has been little improvement. Developing a common, standardized cross-disability tool or protocol for evaluating immediate and long-term intervention impact may help support monitoring and evaluation efforts to further refine and improve training. 
While the review uncovered many examples of disability training, one of the main limitations of the study is that it only highlights published examples of studies, which can leave out unpublished examples. Furthermore, without greater follow-up evaluation or standardization in evaluation, it is difficult to assess the longevity and quality of impact to understand impact of training definitively. On the other hand, this study reveals some adaptable examples of how to integrate disability training into all stages of health worker training and development, which can serve as models for inclusion efforts around the world.

\section{CONCLUSION}

Significant health disparities and poor-quality health care still exist for people with disabilities around the world. Without normalizing disability training as part of high-quality health worker training, there will continue to be limited progress on improving outcomes for people with disabilities. Catalyzing the post-pandemic health systems strengthening efforts to include these evidence-based and effective health worker training on disability can contribute to improved care and outcomes for people with disabilities. 
Appendix A: Details of interventions in included studies

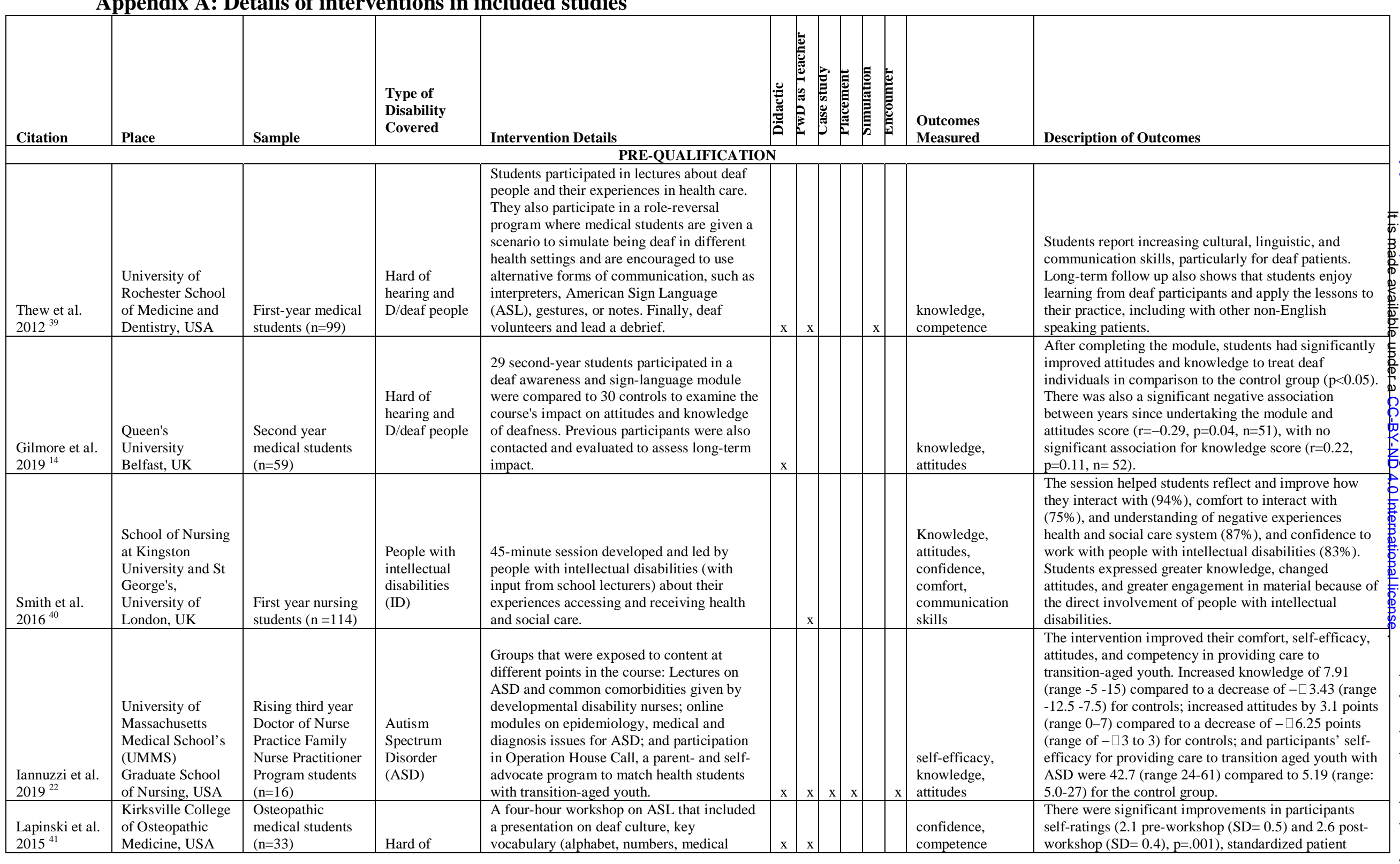




\begin{tabular}{|c|c|c|c|c|c|c|c|c|c|c|c|c|}
\hline & & & $\begin{array}{l}\text { hearing and } \\
\text { D/deaf people }\end{array}$ & $\begin{array}{l}\text { words, etc.) and etiquette, stories from the } \\
\text { Deaf community facilitators, and an } \\
\text { overview of providing health care to deaf } \\
\text { patients. }\end{array}$ & & & & & & & & $\begin{array}{l}\text { scores (pre-workshop encounter (49.6, } \mathrm{SD}=14.3) \text { and } \\
\text { the standardized patient post-workshop encounter }(87.3 \text {, } \\
\mathrm{SD}=10.1), \mathrm{p}<.001) \text {, and knowledge (pre-work-shop } \\
\text { video quiz }(3.6, \mathrm{SD}=3.0) \text { and the post-workshop video } \\
\text { quiz }(16.8, \mathrm{SD}=3.1), \mathrm{p}<.001 .) \text {. }\end{array}$ \\
\hline $\begin{array}{l}\text { Holzinger et } \\
\text { al. } 2020^{42}\end{array}$ & $\begin{array}{l}\text { Medical } \\
\text { University of } \\
\text { Vienna, Austria }\end{array}$ & $\begin{array}{l}\text { Fourth-year dental } \\
\text { students }(\mathrm{n}=154)\end{array}$ & $\begin{array}{l}\text { Patients with } \\
\text { paediatric, } \\
\text { geriatric, } \\
\text { psychiatric, } \\
\text { and } \\
\text { neurological } \\
\text { disorders }\end{array}$ & $\begin{array}{l}\text { Five 2-hour seminars (lecture and small } \\
\text { group case studies) on managing dental } \\
\text { needs of patients led by a dentist and } \\
\text { specialist in the patient population and a } \\
\text { practical course in the community. }\end{array}$ & $\mathrm{x}$ & & $\mathrm{x}$ & $\mathrm{x}$ & & $\mathrm{x}$ & $\begin{array}{l}\text { emotional } \\
\text { reactions, } \\
\text { acceptance, } \\
\text { comfort, attitudes }\end{array}$ & $\begin{array}{l}\text { Emotional reactions to, acceptance of, comfort with, } \\
\text { and attitudes towards people with disabilities improved } \\
\text { after the seminar and the practical course. }\end{array}$ \\
\hline $\begin{array}{l}\text { Saunder and } \\
\text { Knight, } 2017\end{array}$ & $\begin{array}{l}\text { City University } \\
\text { London, UK }\end{array}$ & $\begin{array}{l}\text { Post-grad and } \\
\text { undergraduate } \\
\text { nursing students } \\
(\mathrm{n}=146)\end{array}$ & $\begin{array}{l}\text { people with } \\
\text { intellectual } \\
\text { disabilities }\end{array}$ & $\begin{array}{l}\text { A three-hour, multi-media 'simulation of } \\
\text { simulations' on caring for patients with } \\
\text { intellectual disabilities. The intervention also } \\
\text { includes group discussions and a workbook. }\end{array}$ & $\mathrm{x}$ & & $\mathrm{x}$ & & $\mathrm{x}$ & & $\begin{array}{l}\text { competence, } \\
\text { confidence }\end{array}$ & $\begin{array}{l}\text { Undergraduate students felt their learning, confidence, } \\
\text { and capabilities increased because of the engaging } \\
\text { simulation methods. Overall, } 91 \% \text { of child, } 94 \% \text { of } \\
\text { mental health, and } 84 \% \text { of adult nursing students agreed } \\
\text { that the intervention prepared them for clinical practice } \\
\text { and } 90 \% \text { of students indicated their knowledge } \\
\text { increased. }\end{array}$ \\
\hline $\begin{array}{l}\text { Santoro et al. } \\
2019^{44}\end{array}$ & $\begin{array}{l}\text { Stanford } \\
\text { University, USA }\end{array}$ & $\begin{array}{l}\text { Medical students } \\
\text { in pediatric } \\
\text { clerkship }(\mathrm{n}=24)\end{array}$ & $\begin{array}{l}\text { Intellectual, } \\
\text { language, or } \\
\text { motor } \\
\text { disabilities } \\
\end{array}$ & $\begin{array}{l}\text { A RCT evaluating an intervention that } \\
\text { includes lectures, bedside teaching, and five } \\
\text { clinical learning scenarios compared to a } \\
\text { lecture-only control. Conducted during core } \\
\text { pediatrics rotations. }\end{array}$ & $\mathrm{x}$ & & $\mathrm{x}$ & & & $\mathrm{x}$ & $\begin{array}{l}\text { knowledge, } \\
\text { attitudes }\end{array}$ & $\begin{array}{l}\text { Students' knowledge and attitudes improved more in } \\
\text { the intervention group than the control group (mean test } \\
\text { was } 7.67 \text { to } 8.25 \text { for the intervention group vs. } 7.10 \text { to } \\
7.55 \text { for the control group). }\end{array}$ \\
\hline $\begin{array}{l}\text { Symons et al. } \\
2014^{34}\end{array}$ & $\begin{array}{l}\text { State University } \\
\text { of New York at } \\
\text { Buffalo and } \\
\text { Syracuse Medical } \\
\text { Schools, USA }\end{array}$ & $\begin{array}{l}\text { Medical students } \\
(\mathrm{n}=244)\end{array}$ & $\begin{array}{l}\text { All disability } \\
\text { types }\end{array}$ & $\begin{array}{l}\text { A new medical school curriculum that } \\
\text { combines lectures, small group and } \\
\text { structured encounters with people with } \\
\text { disabilities and their families, clinical } \\
\text { experiences, workshops, and elective options } \\
\text { over four years to improve disability } \\
\text { training. }\end{array}$ & $\mathrm{x}$ & $\mathrm{x}$ & $\mathrm{x}$ & $\mathrm{x}$ & $\mathrm{x}$ & $\mathrm{x}$ & $\begin{array}{l}\text { knowledge, } \\
\text { attitudes }\end{array}$ & $\begin{array}{l}\text { Students who engaged in the curriculum had significant } \\
\text { or nearly significant improvements in knowledge and } \\
\text { attitudes towards people with disabilities compared with } \\
\text { controls. However, men who participated in the clinical } \\
\text { experience had more negative attitudes to people with } \\
\text { disabilities }(\mathrm{p}=0.005) \text {. }\end{array}$ \\
\hline $\begin{array}{l}\text { Perusini et al. } \\
2016^{45}\end{array}$ & $\begin{array}{l}\text { Faculty of } \\
\text { Dentistry, } \\
\text { University of } \\
\text { Toronto, Canada }\end{array}$ & $\begin{array}{l}\text { Senior dental } \\
\text { students }(n=92)\end{array}$ & $\begin{array}{l}\text { People with } \\
\text { disabilities } \\
\text { (PWDs) }\end{array}$ & $\begin{array}{l}\text { Senior dental students participated in a } \\
\text { mandatory clinic placement for people with } \\
\text { disabilities. }\end{array}$ & & & & $\mathrm{x}$ & & $\mathrm{x}$ & attitudes, comfort & $\begin{array}{l}\text { Participants showed that they had improved attitudes } \\
\text { and comfort in treating patients with disabilities. } \\
\text { Previous experience with PWDs was a positive } \\
\text { predictor of comfort in providing care to PWDs. }\end{array}$ \\
\hline $\begin{array}{l}\text { Castro et al. } \\
2018^{46}\end{array}$ & $\begin{array}{l}\text { Federal University } \\
\text { of Triângulo } \\
\text { Mineiro, Brazil }\end{array}$ & $\begin{array}{l}\text { Undergraduate } \\
\text { medicine, nursing, } \\
\text { nutrition, } \\
\text { occupational } \\
\text { therapy, } \\
\text { physiotherapy, } \\
\text { and psychology } \\
\text { students }(\mathrm{n}=12)\end{array}$ & $\begin{array}{l}\text { People with } \\
\text { disabilities }\end{array}$ & $\begin{array}{l}\text { Undergraduate health students participated } \\
\text { in a } 30 \text {-hour course that focused on the } \\
\text { concepts and policies related to disability, } \\
\text { experiences of disability, clinical practice, } \\
\text { and seminar presentations. These topics were } \\
\text { taught through lectures, movies, case studies, } \\
\text { role-play, debates, and discussions. }\end{array}$ & $\mathrm{x}$ & $\mathrm{x}$ & $\mathrm{x}$ & & $\mathrm{x}$ & $\mathrm{x}$ & competence & $\begin{array}{l}\text { The intervention improved students' self-reported core } \\
\text { competencies, particularly in communication, } \\
\text { assessment, provider-patient relationship, practice, and } \\
\text { knowledge of both accessibility and caring for patients } \\
\text { with disabilities. }\end{array}$ \\
\hline $\begin{array}{l}\text { Burrola- } \\
\text { Mendez et al. } \\
2018^{47}\end{array}$ & $\begin{array}{l}\text { University of } \\
\text { Pittsburgh, USA }\end{array}$ & $\begin{array}{l}\text { Graduate students } \\
\text { in rehabilitation } \\
\text { sciences }(\mathrm{n}=6)\end{array}$ & $\begin{array}{l}\text { Wheelchair } \\
\text { users }\end{array}$ & $\begin{array}{l}\text { An 8-module online training course followed } \\
\text { by three-day in-person training for low- } \\
\text { bandwidth areas that increases service } \\
\text { provision for wheelchair users. }\end{array}$ & $\mathrm{x}$ & $\mathrm{x}$ & & $\mathrm{x}$ & & & $\begin{array}{l}\text { knowledge, } \\
\text { competence }\end{array}$ & $\begin{array}{l}\text { Course participants had significant increases in their } \\
\text { competency to deliver wheelchair service provisions, } \\
\text { with an average increase of } 10.84 \pm 5.42, p=0.004 \text {. }\end{array}$ \\
\hline Salama and & College of & Dental students & & A 30-minute DVD presentation on providing & $\mathrm{x}$ & & & & & & knowledge, & The mean \pm SD score of all 150 students' ( 30 students \\
\hline
\end{tabular}




\begin{tabular}{|c|c|c|c|c|c|c|c|c|c|c|c|}
\hline $\begin{array}{l}\text { Al-Balkhi } \\
2020^{18}\end{array}$ & $\begin{array}{l}\text { Dentistry, King } \\
\text { Saud University, } \\
\text { Saudi Arabia }\end{array}$ & $(\mathrm{n}=150)$ & $\begin{array}{l}\text { People with } \\
\text { disabilities }\end{array}$ & oral care for people with disabilities. & & & & & & competence & $\begin{array}{l}\text { from each of the five dental levels) pre-test was } \\
15.46 \pm 2.97 \text {, which increased to } 18.09 \pm 3.37 \text { on the post- } \\
\text { test. }\end{array}$ \\
\hline $\begin{array}{l}\text { Mohebbi et } \\
\text { al. } 2014^{48}\end{array}$ & $\begin{array}{l}\text { Tehran University } \\
\text { of Medical } \\
\text { Sciences, Iran }\end{array}$ & $\begin{array}{l}\text { Senior dental } \\
\text { students }(\mathrm{n}=70)\end{array}$ & $\begin{array}{l}\text { People with } \\
\text { disabilities }\end{array}$ & $\begin{array}{l}\text { The intervention group received training on } \\
\text { providing care for disabled patients through } \\
\text { group discussions, lectures, and hands-on } \\
\text { clinical fluoride varnish/screening for } \\
\text { autistic children. }\end{array}$ & $\mathrm{x}$ & & & $\mathrm{x}$ & $\mathrm{x}$ & $\begin{array}{l}\text { knowledge, } \\
\text { attitudes }\end{array}$ & $\begin{array}{l}\text { The intervention group significantly increased their } \\
\text { attitudes and knowledge after the intervention, even } \\
\text { after controlling for backgrounds and experience with } \\
\text { disability ( }<0.05) \text {. }\end{array}$ \\
\hline $\begin{array}{l}\text { Bailey et al. } \\
2021^{16}\end{array}$ & $\begin{array}{l}\text { Ferris State } \\
\text { University } \\
\text { College of } \\
\text { Pharmacy, USA }\end{array}$ & $\begin{array}{l}\text { First and second- } \\
\text { year pharmacy } \\
\text { students }(n=39)\end{array}$ & $\begin{array}{l}\text { Hard of } \\
\text { hearing and } \\
\text { D/deaf people }\end{array}$ & $\begin{array}{l}\text { A four-class, optional, co-curricular course } \\
\text { was offered to pharmacy students, focusing } \\
\text { on deaf culture, communication, and ASL. } \\
\text { Included time to interact with a deaf } \\
\text { physician over Skype and with deaf/HOH } \\
\text { individuals from the community. }\end{array}$ & $\mathrm{x}$ & $\mathrm{x}$ & & & & $\begin{array}{l}\text { confidence, } \\
\text { communication }\end{array}$ & $\begin{array}{l}\text { Pharmacy students had significant increases in their } \\
\text { confidence to provide care and communicate with } \\
\text { d/Deaf, hard of hearing individuals, and interpreters } \\
\text { after the course. }\end{array}$ \\
\hline $\begin{array}{l}\text { Watmough et } \\
\text { al. } 2014^{27}\end{array}$ & $\begin{array}{l}\text { University of } \\
\text { Liverpool, UK }\end{array}$ & $\begin{array}{l}\text { Third year } \\
\text { medical students } \\
(\mathrm{n}=42)\end{array}$ & $\begin{array}{l}\text { People with } \\
\text { disabilities }\end{array}$ & $\begin{array}{l}\text { A 7-week community placement for third } \\
\text { year medical students, including six full-day, } \\
\text { small-group sessions, a 5-day GP placement } \\
\text { where students clerk with at least two } \\
\text { patients with disabilities, communications } \\
\text { skills development, and visits to community } \\
\text { disability centers }\end{array}$ & $\mathrm{x}$ & & & $\mathrm{x}$ & $\mathrm{x}$ & $\begin{array}{l}\text { knowledge, } \\
\text { comfort }\end{array}$ & $\begin{array}{l}\text { Students better understood disability by participating in } \\
\text { the program and felt that the community center visits, } \\
\text { having patients as educators (through clinical contact), } \\
\text { and workshops throughout the placement had } \\
\text { significant impacts on their knowledge and comfort. }\end{array}$ \\
\hline $\begin{array}{l}\text { Ahmad et al. } \\
2020^{49}\end{array}$ & $\begin{array}{l}\text { Faculty of } \\
\text { Dentistry, } \\
\text { Universiti } \\
\text { Teknologi } \\
\text { MARA, Malaysia }\end{array}$ & $\begin{array}{l}\text { Undergraduate } \\
\text { dental students } \\
(\mathrm{n}=165)\end{array}$ & $\begin{array}{l}\text { Children with } \\
\text { visual } \\
\text { impairments }\end{array}$ & $\begin{array}{l}\text { Dental students (years 1-5) received a lecture } \\
\text { on management of individuals with visual } \\
\text { impairments followed by a participating in a } \\
\text { clinic where each dental student was } \\
\text { assigned to manage of a child with visual } \\
\text { impairment at a special education school as } \\
\text { part of a dedicated module. }\end{array}$ & $\mathrm{x}$ & & & $\mathrm{x}$ & & $\begin{array}{l}\text { knowledge, } \\
\text { competence, } \\
\text { behaviour, } \\
\text { attitudes, learning }\end{array}$ & $\begin{array}{l}\text { Students positively improved their knowledge, } \\
\text { professionalism, behaviour, attitudes, and learning by } \\
\text { participating in the clinic. }\end{array}$ \\
\hline $\begin{array}{l}\text { Crane et al. } \\
2020^{50}\end{array}$ & $\begin{array}{l}\text { Ohio State } \\
\text { University } \\
\text { Wexner Medical } \\
\text { Center, USA } \\
\end{array}$ & $\begin{array}{l}\text { Third-year } \\
\text { medical students } \\
(\mathrm{n}=169)\end{array}$ & $\begin{array}{l}\text { People with } \\
\text { disabilities } \\
\text { (mobility, } \\
\text { sensory, } \\
\text { cognitive) } \\
\end{array}$ & $\begin{array}{l}\text { Medical students participated in a patient } \\
\text { encounter with a PWD where they were } \\
\text { asked to complete a brief social and medical } \\
\text { history. }\end{array}$ & & & & & $\mathrm{x}$ & $\begin{array}{l}\text { confidence, } \\
\text { competence, and } \\
\text { comfort }\end{array}$ & $\begin{array}{l}\text { Students initially showed significant improvements in } \\
\text { confidence, skills, and comfort. A year later, 35\% of } \\
\text { participants were surveyed and found these benefits } \\
\text { were sustained in most students, though many felt the } \\
\text { need for more training and exposure. }\end{array}$ \\
\hline $\begin{array}{l}\text { Tai et al. } \\
2018^{51}\end{array}$ & $\begin{array}{l}\text { University of } \\
\text { Melbourne, } \\
\text { Australia }\end{array}$ & $\begin{array}{l}\text { Final year } \\
\text { graduate } \\
\text { audiology } \\
\text { students }(n=15)\end{array}$ & $\begin{array}{l}\text { Hard of } \\
\text { hearing and } \\
\text { D/deaf people }\end{array}$ & $\begin{array}{l}\text { Students had a clinical encounter filmed and } \\
\text { re-played during a clinical communication } \\
\text { education session to see their perspectives on } \\
\text { their clinical communication skills. }\end{array}$ & & & & & $\mathrm{x}$ & communication & $\begin{array}{l}\text { Audiology students demonstrated that they were able to } \\
\text { reflect on critical communication areas and be more } \\
\text { attentive to patient's needs, understanding, and } \\
\text { questions. }\end{array}$ \\
\hline $\begin{array}{l}\text { Ozkan et al. } \\
2020^{52}\end{array}$ & $\begin{array}{l}\text { Faculty of Health } \\
\text { Sciences, State } \\
\text { University in } \\
\text { Turkey }\end{array}$ & $\begin{array}{l}\text { Third-year } \\
\text { nursing students } \\
(\mathrm{n}=70)\end{array}$ & $\begin{array}{l}\text { People with } \\
\text { intellectual } \\
\text { disabilities }\end{array}$ & $\begin{array}{l}\text { Students participated in a clinical practice } \\
\text { for three weeks ( } 2.5 \text { days per week) in } 12 \\
\text { different fields- one of them being in a } \\
\text { special education school. The experimental } \\
\text { group went to a clinic that served special } \\
\text { education schools and were exposed to case } \\
\text { studies, nursing care examples, and videos } \\
\text { care for people with disabilities. }\end{array}$ & $\mathrm{x}$ & & & $\mathrm{x}$ & & $\begin{array}{l}\text { knowledge, } \\
\text { competence, } \\
\text { attitudes }\end{array}$ & $\begin{array}{l}\text { After the placement, the experimental group had higher } \\
\text { total attitudes to people with disabilities ( } 172.02 \pm 14.59 \\
\text { pre-test to } \\
177.54 \pm 13.95 \text { post-test) compared to the control group } \\
(176.31 \pm 16.25 \text { pre-test to } 171.97 \pm 13.69 \text { post-test). } \\
\text { Students also noted that they improved their attitudes, } \\
\text { knowledge, and skills for caring for people with } \\
\text { intellectual disabilities. }\end{array}$ \\
\hline
\end{tabular}




\begin{tabular}{|c|c|c|c|c|c|c|c|c|c|c|c|}
\hline $\begin{array}{l}\text { Sinai et al. } \\
2013^{53}\end{array}$ & $\begin{array}{l}\text { University } \\
\text { College London, } \\
\text { UK }\end{array}$ & $\begin{array}{l}\text { Undergraduate } \\
\text { medical students } \\
(\mathrm{n}=387)\end{array}$ & $\begin{array}{l}\text { People with } \\
\text { intellectual } \\
\text { disabilities }\end{array}$ & $\begin{array}{l}\text { A 14-week course taught undergraduate } \\
\text { medical students about training patients with } \\
\text { intellectual disabilities. As part of the block, } \\
\text { participants did a three-week specialist } \\
\text { placement on learning disability services. }\end{array}$ & $\mathrm{x}$ & & $\mathrm{x}$ & $\mathrm{x}$ & & $\begin{array}{l}\text { knowledge, } \\
\text { attitudes }\end{array}$ & $\begin{array}{l}\text { Students basic knowledge significantly improved after } \\
\text { the intervention }(\mathrm{p}<0.001) \text { and improved their attitudes, } \\
\text { though not significantly. }\end{array}$ \\
\hline $\begin{array}{l}\text { Abdi and } \\
\text { Metcalf } 2020\end{array}$ & $\begin{array}{l}\text { Cardiff } \\
\text { University, UK }\end{array}$ & $\begin{array}{l}\text { Fourth-year } \\
\text { undergraduate } \\
\text { medical students } \\
(\mathrm{n}=110)\end{array}$ & $\begin{array}{l}\text { People with } \\
\text { intellectual } \\
\text { disabilities }\end{array}$ & $\begin{array}{l}\text { Medical students participated in a mandatory } \\
\text { communication skills session that included } \\
\text { case studies and simulated patients. This was } \\
\text { run by the Speech Language Therapy team } \\
\text { three times during the academic year. }\end{array}$ & $\mathrm{x}$ & & $\mathrm{x}$ & & $\mathrm{x}$ & $\begin{array}{l}\text { confidence, } \\
\text { attitudes }\end{array}$ & $\begin{array}{l}\text { Mean scores improved from } 115 \text { ( } \mathrm{SD}=14.5) \text { to } 122 \\
\text { ( } \mathrm{SD}=17.2) \text {, or a mean difference of } 6.92 \text { (C.I: } 4.69 \text {, } \\
\text { 9.16), which was statistically significant. Participants } \\
\text { also noted their confidence on treating patients with } \\
\text { disabilities also improved. }\end{array}$ \\
\hline $\begin{array}{l}\text { Shields et al. } \\
2013^{54}\end{array}$ & $\begin{array}{l}\text { Department of } \\
\text { Physiotherapy, La } \\
\text { Trobe University, } \\
\text { Australia }\end{array}$ & $\begin{array}{l}\text { First- and second- } \\
\text { year } \\
\text { physiotherapy } \\
\text { students }(\mathrm{n}=17)\end{array}$ & $\begin{array}{l}\text { People with } \\
\text { Down } \\
\text { syndrome }\end{array}$ & $\begin{array}{l}\text { Students participated in a } 10 \text {-week, twice } \\
\text { weekly community placement at a } \\
\text { gymnasium for adolescents with Down } \\
\text { syndrome. There, participants were expected } \\
\text { to enact a progressive resistance training } \\
\text { program. }\end{array}$ & & $x$ & & $\mathrm{x}$ & & $\begin{array}{l}\text { communication, } \\
\text { comfort, } \\
\text { knowledge }\end{array}$ & $\begin{array}{l}\text { Physiotherapy students improved their clinical skills, } \\
\text { professional skills, and understanding of young people } \\
\text { with disabilities. }\end{array}$ \\
\hline $\begin{array}{l}\text { Hensel et al. } \\
2015^{28}\end{array}$ & $\begin{array}{l}\text { University- } \\
\text { affiliated } \\
\text { wilderness } \\
\text { children's camp, } \\
\text { USA }\end{array}$ & $\begin{array}{l}\text { Junior-level BSN } \\
\text { students }(n=9)\end{array}$ & $\begin{array}{l}\text { Children with } \\
\text { intellectual } \\
\text { impairments } \\
\text { or wheelchair } \\
\text { users }\end{array}$ & $\begin{array}{l}\text { Nursing students went to one of two } \\
\text { weekend-long fall camps for children with } \\
\text { disabilities aged 8-18. }\end{array}$ & & & & $\mathrm{x}$ & & $\begin{array}{l}\text { knowledge, } \\
\text { comfort, attitudes }\end{array}$ & $\begin{array}{l}\text { Students found the placement challenging, but overall } \\
\text { improved their confidence and competency to provide } \\
\text { care to children with disabilities/medical complexities. } \\
\text { They also developed more positive attitudes towards } \\
\text { caring for patients with disability. }\end{array}$ \\
\hline $\begin{array}{l}\text { Watkins and } \\
\text { Colgate } 2016\end{array}$ & $\begin{array}{l}\text { Cardiff } \\
\text { University, UK }\end{array}$ & $\begin{array}{l}\text { Medical students } \\
(\mathrm{n}=45)\end{array}$ & $\begin{array}{l}\text { People with } \\
\text { intellectual } \\
\text { disabilities }\end{array}$ & $\begin{array}{l}\text { A self-advocacy agency, Cardiff People } \\
\text { First, ran a one-hour video and ice breaker } \\
\text { activity. Subsequently, students assessed a } \\
\text { simulated patient played by an actor with an } \\
\text { intellectual disability. Students then } \\
\text { participated in a focus group discussions. }\end{array}$ & $\mathrm{x}$ & $\mathrm{x}$ & & & $\mathrm{x}$ & $\begin{array}{l}\text { knowledge, } \\
\text { attitudes }\end{array}$ & $\begin{array}{l}\text { Students attitudes improved significantly from a mean } \\
\text { score of } 13.7(\mathrm{SD}=1.29) \text { to } 10.52(\mathrm{SD}=1.12)(\mathrm{a} \text { mean } \\
\text { difference of } 3.17(95 \% \mathrm{CI}=2.41-3.94), \mathrm{p}<0.001) . \\
\text { Knowledge also improved significantly from a mean of } \\
14.84 \text { pre-intervention to } 10.65 \text { post-intervention (mean } \\
\text { difference } 95 \% \mathrm{CI}=4.22(3.3-5.14), \mathrm{p}<0.001)\end{array}$ \\
\hline $\begin{array}{l}\text { Forsgren et } \\
\text { al. } 2017^{56}\end{array}$ & $\begin{array}{l}\text { University of } \\
\text { Gothenburg, } \\
\text { Sweden }\end{array}$ & $\begin{array}{l}\text { Fourth-year } \\
\text { medical students } \\
(\mathrm{n}=69)\end{array}$ & $\begin{array}{l}\text { People with } \\
\text { acquired } \\
\text { communicatio } \\
\text { n disorders }\end{array}$ & $\begin{array}{l}\text { All students participated in a } 45 \text {-minute } \\
\text { mandatory lecture on acquired } \\
\text { communication disorders. } 36 \text { students then } \\
\text { participated in a } 2.5 \text {-hour long workshop } \\
\text { with a simulated patient to practice } \\
\text { communication skills, and } 15 \text { were video } \\
\text { recorded during the simulation pre- and post- } \\
\text { workshop. }\end{array}$ & $\mathrm{x}$ & & $\mathrm{x}$ & & $\mathrm{x}$ & $\begin{array}{l}\text { knowledge, } \\
\text { attitudes, } \\
\text { communication }\end{array}$ & $\begin{array}{l}\text { Students who participated in the workshop had } \\
\text { significantly higher post-test results than students who } \\
\text { only participated in the lecture, though all groups had } \\
\text { knowledge and communication improvements. There } \\
\text { was also a statistically significant increase in perceived } \\
\text { confidence from pre-lecture }(\mathrm{m}=13) \text { to post-lecture } \\
(\mathrm{m}=18.9, \mathrm{p}=0.001) \text { as well as from pre- } \\
\text { workshop }(\mathrm{m}=15.3) \text { to post-workshop }(\mathrm{m}=21.9, \\
\mathrm{z}=5.168, \mathrm{p}=0.001) \text {. Video recordings supportive } \\
\text { strategies were used more significantly post-workshop. }\end{array}$ \\
\hline $\begin{array}{l}\text { McIntosh et } \\
\text { al. } 2018^{57}\end{array}$ & $\begin{array}{l}\text { Ball State } \\
\text { University School } \\
\text { of Nursing, USA }\end{array}$ & $\begin{array}{l}\text { Senior nursing } \\
\text { students }(\mathrm{n}=84)\end{array}$ & $\begin{array}{l}\text { Autism } \\
\text { Spectrum } \\
\text { Disorder }\end{array}$ & $\begin{array}{l}\text { Students were exposed to Autism through } \\
\text { lectures, case studies, a documentary video, } \\
\text { and description of the simulation prior to the } \\
\text { study, which was an encounter with a } \\
\text { standardized patient. In the encounter, a 14- } \\
\text { year-old boy with a fractured wrist was } \\
\text { admitted to the emergency department. } \\
\text { Students were expected to assess the patient } \\
\text { and associated behavioral and emotional } \\
\text { symptoms. }\end{array}$ & $\mathrm{x}$ & & $\mathrm{x}$ & & $\mathrm{x}$ & $\begin{array}{l}\text { knowledge, } \\
\text { competence }\end{array}$ & $\begin{array}{l}87 \% \text { of students indicated that they used } \\
\text { communications strategies learned, while the rest } \\
\text { remained neutral. } 86 \% \text { also thought the supplemental } \\
\text { information provided ahead of time supported their } \\
\text { learning. Overall, the simulation and pre-requisite } \\
\text { classroom instruction improved their knowledge of } \\
\text { ASD. }\end{array}$ \\
\hline
\end{tabular}




\begin{tabular}{|c|c|c|c|c|c|c|c|c|c|c|c|c|}
\hline $\begin{array}{l}\text { Woodard et } \\
\text { al. } 2012^{58}\end{array}$ & $\begin{array}{l}\text { University of } \\
\text { South Florida } \\
\text { Health, Morsani } \\
\text { College of } \\
\text { Medicine (USF), } \\
\text { USA }\end{array}$ & $\begin{array}{l}\text { Primary care } \\
\text { clerkship for } \\
\text { third-year } \\
\text { undergraduate } \\
\text { medical students } \\
(\mathrm{n}=245)\end{array}$ & $\begin{array}{l}\text { People with } \\
\text { physical, } \\
\text { sensory, or } \\
\text { intellectual } \\
\text { disabilities }\end{array}$ & $\begin{array}{l}\text { Students participated in a 12-week Primary } \\
\text { Care and Special Populations clerkship that } \\
\text { included a special 6-week module on } \\
\text { disability, half-day classroom sessions, } \\
\text { online modules, and community site visits } \\
\text { twice a week. Classroom sessions included } \\
\text { simulations, case studies, and } \\
\text { communication and exam technique advice } \\
\text { from individuals with disabilities. }\end{array}$ & $\mathrm{x}$ & $\mathrm{x}$ & $\mathrm{x}$ & $\mathrm{x}$ & $\mathrm{x}$ & $\mathrm{x}$ & $\begin{array}{l}\text { knowledge, } \\
\text { competence, } \\
\text { comfort }\end{array}$ & $\begin{array}{l}\text { Students significantly improved their knowledge of, } \\
\text { attitudes, and comfort of working with people with } \\
\text { disabilities by a mean score of } 2.36,4.20 \text {, and } 15.00 \text {, } \\
\text { respectively, after completing the clerkship program } \\
\text { (p<0.001 for all). }\end{array}$ \\
\hline $\begin{array}{l}\text { Jones et al. } \\
2015^{59}\end{array}$ & $\begin{array}{l}\text { Queen's } \\
\text { University, } \\
\text { Canada }\end{array}$ & $\begin{array}{l}\text { Medicine }(n=100) \\
\text { nursing }(n=93) \\
\text { clinical } \\
\text { psychology }(n=6) \\
\text { and rehabilitation } \\
\text { students }(n=133) \\
\text { (total } n=332)\end{array}$ & $\begin{array}{l}\text { People with } \\
\text { intellectual } \\
\text { disabilities }\end{array}$ & $\begin{array}{l}\text { Students participated in a three-part course } \\
\text { that consisted of an e-learning and case } \\
\text { models pre-requisite prior to a day-long } \\
\text { course that consisted of group lectures, } \\
\text { multidisciplinary experiential learning, and } \\
\text { inter-professional team-based learning. }\end{array}$ & $\mathrm{x}$ & $\mathrm{x}$ & $\mathrm{x}$ & & & & $\begin{array}{l}\text { knowledge, } \\
\text { competence, } \\
\text { attitudes }\end{array}$ & $\begin{array}{l}\text { Across all disciplines, there was a significant difference } \\
(\mathrm{p}<0.01) \text { in mean percentage correct between pre- } \\
\text { course }(\mathrm{M}=0.64, \mathrm{SD}=0.25) \text { and post-course }(\mathrm{M}=0.70 \text {, } \\
\mathrm{SD}=0.25) \text { skills scores, of which nurses had the most } \\
\text { significant improvements. Knowledge improved pre- } \\
\text { course }(\mathrm{M}=0.51, \mathrm{SD}=0.22) \text { and post-course }(\mathrm{M}=0.64 \text {, } \\
\mathrm{SD}=0.23) \text { significantly }(\mathrm{p}<0.01) \text {, though this was } \\
\text { independently significant for medical, rehabilitation, } \\
\text { and nursing students. There were no statistically } \\
\text { significant differences in attitude. }\end{array}$ \\
\hline $\begin{array}{l}\text { Beverly and } \\
\text { Wooster } 2018 \\
60\end{array}$ & $\begin{array}{l}\text { University of } \\
\text { South Alabama, } \\
\text { USA }\end{array}$ & $\begin{array}{l}\text { Speech language } \\
\text { pathology, } \\
\text { occupational } \\
\text { therapy, and } \\
\text { physiotherapy } \\
\text { graduate } \\
\text { students }(\mathrm{n}=9) \\
\end{array}$ & $\begin{array}{l}\text { Autism } \\
\text { Spectrum } \\
\text { Disorder } \\
\end{array}$ & $\begin{array}{l}\text { A one-credit online course and day-long } \\
\text { conference included lectures, panel } \\
\text { discussions, group problem solving, and } \\
\text { parent perspectives. Content was focused on } \\
\text { inter-professional education and diagnosis } \\
\text { and co-morbidities of ASD. }\end{array}$ & $\mathrm{x}$ & & $\mathrm{x}$ & & & & $\begin{array}{l}\text { knowledge, } \\
\text { competence, } \\
\text { attitudes }\end{array}$ & $\begin{array}{l}\text { There were significant increases in participants basic } \\
\text { ASD knowledge after the course }(\mathrm{p}<0.05) \text {. Participants } \\
\text { were more aware of their role and that of their team } \\
\text { members in the screening and assessment of ASD. }\end{array}$ \\
\hline $\begin{array}{l}\text { Coret et al. } \\
2018^{61}\end{array}$ & $\begin{array}{l}\text { Michael G. } \\
\text { Degroote School } \\
\text { of Medicine, } \\
\text { McMaster } \\
\text { University, } \\
\text { Canada }\end{array}$ & $\begin{array}{l}\text { First-year medical } \\
\text { students }(n=27)\end{array}$ & $\begin{array}{l}\text { Intellectual/de } \\
\text { velopmental } \\
\text { disabilities } \\
\text { (IDD) }\end{array}$ & $\begin{array}{l}\text { Control and intervention groups participated } \\
\text { in a baseline introductory video about } \\
\text { healthcare and communication skills. After } \\
\text { the video, the intervention group were shown } \\
\text { videos of individuals and families of } \\
\text { individuals with IDD who spoke about their } \\
\text { lives, challenges, and perspectives on health } \\
\text { care and participated in a reflective activity. } \\
\text { Controls were only given a post-intro video } \\
\text { quiz. Both groups participated in four } \\
\text { clinical encounters and their skills were } \\
\text { assessed by a final-year student. }\end{array}$ & $\mathrm{x}$ & & & & & $\mathrm{x}$ & $\begin{array}{l}\text { comfort, } \\
\text { confidence, and } \\
\text { competence }\end{array}$ & $\begin{array}{l}\text { Students communication skills assessment mean rating } \\
\text { scores were higher for students in the narrative group } \\
\text { relative to the control group for self-ratings mean }[\mathrm{SD}] \\
4.49[0.73] \text { vs. } 4.37[0.69]) \text {, patient educator } \\
\text { ratings( } 5.43[0.52] \text { vs. } 5.33[0.76]) \text {, and senior medical } \\
\text { students }(5.01[0.69] \text { vs. } 4.75[0.79]) \text {. Similarly, } \\
\text { students' qualitative evaluations demonstrated improved } \\
\text { communication, comfort, confidence, adaptability, and } \\
\text { learning from the methods used in the intervention } \\
\text { group. }\end{array}$ \\
\hline $\begin{array}{l}\text { Jain et al. } \\
2013^{62}\end{array}$ & $\begin{array}{l}\text { Department of } \\
\text { Family Medicine, } \\
\text { Lehigh Valley } \\
\text { Health Network, } \\
\text { USA }\end{array}$ & $\begin{array}{l}\text { Third- and fourth- } \\
\text { year medical } \\
\text { students }(n=44)\end{array}$ & $\begin{array}{l}\text { Wheelchair } \\
\text { users }\end{array}$ & $\begin{array}{l}\text { Two patients with disabilities were trained to } \\
\text { teach medical students about disability. In an } \\
\text { hour-long session, they described their daily } \\
\text { routines and experiences interacting with } \\
\text { health care for medical students, followed by } \\
\text { Q and A. Students who participated in the } \\
\text { session were asked questions about } \\
\text { awareness, sensitivity, and competency. }\end{array}$ & $\mathrm{x}$ & $\mathrm{x}$ & & & & & $\begin{array}{l}\text { comfort, } \\
\text { knowledge, } \\
\text { competency, } \\
\text { communication }\end{array}$ & $\begin{array}{l}\text { Respondents qualitative responses suggested greater } \\
\text { competency and awareness and sensitivity after the } \\
\text { intervention, particularly since prior to the training } 89 \% \\
\text { felt uncomfortable interacting with patients with } \\
\text { disabilities. Overall, } 98 \% \text { stated the program was } \\
\text { beneficial. }\end{array}$ \\
\hline Karl et al. & University at & Third-year & & Third-year medical students participating in & & & & & & $\mathrm{x}$ & communication, & Students felt that the encounter highlighted gaps in \\
\hline
\end{tabular}




\begin{tabular}{|c|c|c|c|c|c|c|c|c|c|c|c|c|}
\hline $2013^{63}$ & $\begin{array}{l}\text { Buffalo School of } \\
\text { Medicine and } \\
\text { Biomedical } \\
\text { Sciences, USA }\end{array}$ & $\begin{array}{l}\text { medical students } \\
(\mathrm{n}=144)\end{array}$ & $\begin{array}{l}\text { People with } \\
\text { disabilities } \\
\text { (mostly } \\
\text { people with } \\
\text { developmenta } \\
1 \text { disabilities) }\end{array}$ & $\begin{array}{l}\text { a family medicine clerkship program } \\
\text { engaged in a day-long clinical experience at } \\
\text { a family practice serving people with } \\
\text { disabilities. Students observed and assisted } \\
\text { the staff and then were asked to write a } \\
\text { reflection. Students had previously done } \\
\text { lectures, small group encounters of people } \\
\text { with disabilities and their families, and other } \\
\text { workshops. }\end{array}$ & & & & & & & $\begin{array}{l}\text { comfort, } \\
\text { attitudes, } \\
\text { knowledge }\end{array}$ & $\begin{array}{l}\text { communication skills, as well as increasing comfort in } \\
\text { caring for people with disabilities. The program also } \\
\text { highlighted quality and standard of care issues for } \\
\text { people with disabilities and that ones' disability can } \\
\text { wrongly be attributed as the cause of the issue, when it's } \\
\text { not. Students also noted issues with the organizational } \\
\text { structure of the medical facility and environmental and } \\
\text { technical accommodations necessary for support. }\end{array}$ \\
\hline $\begin{array}{l}\text { Thomas et al. } \\
2014^{64}\end{array}$ & $\begin{array}{l}\text { University } \\
\text { College London, } \\
\text { UK }\end{array}$ & $\begin{array}{l}\text { 4th year } \\
\text { undergraduate } \\
\text { medical students } \\
(\mathrm{n}=47)\end{array}$ & $\begin{array}{l}\text { People with } \\
\text { intellectual } \\
\text { disabilities }\end{array}$ & $\begin{array}{l}\text { All participants had attended a 3-hour lecture } \\
\text { on ID, session by a speech and language } \\
\text { therapist, group work, watching a } \\
\text { communication DVD, and basic Makaton } \\
\text { training (a simplified British Sign } \\
\text { Language). The second session involved } \\
\text { students rotating through four stations in } \\
\text { groups of four to work with simulated } \\
\text { patients with ID. }\end{array}$ & $\mathrm{x}$ & & & & $\mathrm{x}$ & & $\begin{array}{l}\text { competence, } \\
\text { comfort, } \\
\text { communication }\end{array}$ & $\begin{array}{l}\text { There was significant improvement in medical students' } \\
\text { perceived skills }(\mathrm{p}=0.002) \text {, comfort level }(\mathrm{p}=0.004) \text {, and } \\
\text { type of clinical approach }(\mathrm{p}=0.003) \text { in managing } \\
\text { patients with no disability. Furthermore, significantly } \\
\text { improved scores for all three factors were obtained by } \\
\text { medical students regarding patients with mild and } \\
\text { severe disability levels ( }<0.001) \text {, with even greater } \\
\text { benefits for managing severe disabilities compared to } \\
\text { mild. }\end{array}$ \\
\hline $\begin{array}{l}\text { Saldert et al. } \\
2016^{31}\end{array}$ & $\begin{array}{l}\text { University of } \\
\text { Gothenburg, } \\
\text { Sweden }\end{array}$ & $\begin{array}{l}\text { Medical students } \\
(\mathrm{n}=59,33 \text { only } \\
\text { lecture, } 26 \text { lecture } \\
\& \text { workshop })\end{array}$ & $\begin{array}{l}\text { People with } \\
\text { speech } \\
\text { language } \\
\text { disorders }\end{array}$ & $\begin{array}{l}\text { Students attended a } 45 \text { min mandatory } \\
\text { lecture on different forms of speech } \\
\text { disorders. A subset of students attended a } \\
\text { mandatory } 2.5 \text {-hour mixed format workshop } \\
\text { two weeks later, led by speech language } \\
\text { pathologists, involving role play, a lecture on } \\
\text { communication strategies, and discussions. }\end{array}$ & $\mathrm{x}$ & & $\mathrm{x}$ & & $\mathrm{x}$ & & $\begin{array}{l}\text { knowledge, } \\
\text { confidence, } \\
\text { competence, } \\
\text { attitudes }\end{array}$ & $\begin{array}{l}\text { Students from both the lecture and the workshop } \\
\text { reported significantly increased knowledge }(\mathrm{p}=0.001) \\
\text { and how to support communication in speech-language } \\
\text { disorders. Only the workshop group also displayed a } \\
\text { statistically significant increase in ability to suggest } \\
\text { suitable communications strategies }(\mathrm{p}=0.001) \text { and } \\
\text { change in attitudes }(\mathrm{p}=0.001) \text {. }\end{array}$ \\
\hline $\begin{array}{l}\text { Baylor et al. } \\
2019^{65}\end{array}$ & $\begin{array}{l}\text { University of } \\
\text { Washington, USA }\end{array}$ & $\begin{array}{l}\text { Second-year } \\
\text { medical students } \\
(\mathrm{n}=224)\end{array}$ & $\begin{array}{l}\text { People with } \\
\text { communicatio } \\
\text { n disorders }\end{array}$ & $\begin{array}{l}\text { Students received the FRAME training, } \\
\text { which looks at five key areas of } \\
\text { communication skills, including } \\
\text { familiarizing patient communication } \\
\text { preferences, reducing rate, assisting patients, } \\
\text { mixing methods, and respecting autonomy. } \\
\text { Students participated in a 20-minute module } \\
\text { on key characteristics of communications } \\
\text { disorders, a 2-hour in-person session and } \\
\text { practice strategies and simulated patients } \\
\text { with aphasia and dysarthria. }\end{array}$ & $\mathrm{x}$ & & & & $\mathrm{x}$ & & $\begin{array}{l}\text { knowledge, self- } \\
\text { efficacy, } \\
\text { communication, } \\
\text { confidence }\end{array}$ & $\begin{array}{l}\text { Knowledge scores significantly increased from a mean } \\
\text { of } 4.96 \text { pre-training }(\mathrm{SD}=1.1) \text { to } 7.23 \text { post-training } \\
(\mathrm{SD}=1.8)(\mathrm{p}=0.000) \text {, while self-efficacy improved } \\
\text { significantly across all measures ( } \mathrm{p}=0.000 \text { ). Students } \\
\text { also significantly improved their communications skills, } \\
\text { particularly in diagnosing and familiarizing with patient } \\
\text { communication preferences (except for reducing rate in } \\
\text { the dysarthria scenario). }\end{array}$ \\
\hline $\begin{array}{l}\text { Lynch et al. } \\
2019^{21}\end{array}$ & $\begin{array}{l}\text { Medical School in } \\
\text { Ireland }\end{array}$ & $\begin{array}{l}\text { Undergraduate, } \\
\text { graduate entry, } \\
\text { and international } \\
\text { medical students } \\
\text { in second or third } \\
\text { year }(\mathrm{n}=320)\end{array}$ & $\begin{array}{l}\text { People with } \\
\text { disabilities }\end{array}$ & $\begin{array}{l}\text { Students taking a mandatory } 12 \text { week } \\
\text { 'Understanding Disability' module in their } \\
\text { course. This involved weekly lectures, } \\
\text { applied anatomy laboratory sessions, a day } \\
\text { visit to a national rehabilitation hospital, } \\
\text { practice taking patient history in } \\
\text { rehabilitation programs, and case studies. }\end{array}$ & $\mathrm{x}$ & $\mathrm{x}$ & $\mathrm{x}$ & $\mathrm{x}$ & & $\mathrm{x}$ & $\begin{array}{l}\text { competency, } \\
\text { attitude }\end{array}$ & $\begin{array}{l}\text { An overall positive effect across the module was } \\
\text { observed with significantly improved measurements } \\
\text { from pre-intervention to post-intervention across the } 4 \\
\text { variables, anxiety ( }<0.0125) \text {, attitude and competency } \\
\text { ( }<0.001) \text {, and empathy ( } \mathrm{p}=0.03 \text { ). }\end{array}$ \\
\hline
\end{tabular}




\begin{tabular}{|c|c|c|c|c|c|c|c|c|c|c|c|}
\hline $\begin{array}{l}\text { Garavatti et } \\
\text { al. } 2018^{35}\end{array}$ & $\begin{array}{l}\text { University of } \\
\text { Central Florida, } \\
\text { USA }\end{array}$ & $\begin{array}{l}\text { 2nd year medical } \\
\text { student }(\mathrm{n}=20) \text {, } \\
\text { final year Doctor } \\
\text { of Physical } \\
\text { Therapy Students } \\
(\mathrm{n}=20)\end{array}$ & $\begin{array}{l}\text { People with } \\
\text { disabilities }\end{array}$ & $\begin{array}{l}\text { Medical and physical therapy students in the } \\
\text { intervention group went through an } \\
\text { interprofessional education (IPE) clinical } \\
\text { experience that included an encounter with a } \\
\text { disabled patient. } 20 \text { students attended a } 3.5- \\
\text { hour IPE clinical session, focused on } \\
\text { knowledge and interacting with individuals } \\
\text { with disabilities through an orientation, } \\
\text { performing a neurological examination, and } \\
\text { group discussion. The control group (n=20) } \\
\text { did not attend the IPE clinical session. }\end{array}$ & $\mathrm{x}$ & & & & $\mathrm{x}$ & $\begin{array}{l}\text { knowledge, } \\
\text { attitudes, } \\
\text { competence }\end{array}$ & $\begin{array}{l}\text { Before the intervention, interactions of disabled persons } \\
\text { was significantly higher in the experimental group and } \\
\text { higher in medical students. Pre and posttest scores for } \\
\text { the intervention group revealed a statistically significant } \\
(\text { p }<0.001) \text {, with improved comfort levels in } 5 \text { of } 6 \\
\text { subscales. However, mean scores did not differ } \\
\text { significantly between control and experimental groups } \\
\text { (p>0.05). Both medical and physical therapy students } \\
\text { reported greater comfort levels and perception attitudes } \\
\text { in working with patients with disabilities after the } \\
\text { intervention. }\end{array}$ \\
\hline $\begin{array}{l}\text { Saunder and } \\
\text { Berridge } \\
2015^{66}\end{array}$ & $\begin{array}{l}\text { City University } \\
\text { London, UK }\end{array}$ & $\begin{array}{l}\text { Undergraduate } \\
\text { and post-graduate } \\
\text { adult, child, and } \\
\text { mental health } \\
\text { nurses }(\mathrm{n}=182)\end{array}$ & $\begin{array}{l}\text { People with } \\
\text { intellectual } \\
\text { disabilities }\end{array}$ & $\begin{array}{l}\text { Nursing students participated in a 3-hour } \\
\text { session on education around people with } \\
\text { intellectual disabilities. This involved } \\
\text { 'Shareville', a virtual simulation/software } \\
\text { allowing for navigation of real-life scenarios } \\
\text { of individuals with disabilities. Group } \\
\text { discussions occurred prior to and after } \\
\text { Shareville utilization. }\end{array}$ & $\mathrm{x}$ & & & $x$ & & $\begin{array}{l}\text { knowledge, } \\
\text { confidence }\end{array}$ & $\begin{array}{l}\text { Students reported that the Shareville scenarios were } \\
\text { sufficiently real and generally had a positive evaluation, } \\
\text { citing improved confidence, knowledge, clinical } \\
\text { practice, and decision making. Lecturers identified } \\
\text { strengths in the blended learning and collaborative } \\
\text { teaching approach. }\end{array}$ \\
\hline $\begin{array}{l}\text { Sheepway et } \\
\text { al. } 2014^{30}\end{array}$ & $\begin{array}{l}\text { Faculty of health } \\
\text { sciences, Australia }\end{array}$ & $\begin{array}{l}\text { Third year } \\
\text { undergraduate } \\
\text { speech-language } \\
\text { pathology } \\
\text { students }(\mathrm{n}=73)\end{array}$ & $\begin{array}{l}\text { People with } \\
\text { communicatio } \\
\mathrm{n} \\
\text { impairments }\end{array}$ & $\begin{array}{l}\text { Third year speech language pathology } \\
\text { students participated in three clinical } \\
\text { placements to develop clinical competency } \\
\text { over } 12 \text { months. The intervention sought to } \\
\text { examine the impact of the sequence of } \\
\text { placements, setting, and intensity of } \\
\text { placements. }\end{array}$ & & & $\mathrm{x}$ & & $\mathrm{x}$ & competence & $\begin{array}{l}\text { Students performed better on the } 11 \text { competencies } \\
\text { measured in COMPASS with each subsequent clinical } \\
\text { encounter. Mean competency scores for placement } 1 \\
400.96(\mathrm{SD}=57.404) \text {, placement } 2451.55 \text { ( } \mathrm{SD}=81.116 \text { ), } \\
\text { and placement } 3510.53 \text { ( } \mathrm{SD}=92.874) \text {. Students in child } \\
\text { placements were found to achieve greater growth in } \\
\text { overall competency than those in adult placements. }\end{array}$ \\
\hline $\begin{array}{l}\text { Steedman et } \\
\text { al. } 2012^{19}\end{array}$ & $\begin{array}{l}\text { Queen's } \\
\text { University, } \\
\text { Canada }\end{array}$ & $\begin{array}{l}\text { Second year } \\
\text { medical students } \\
(\mathrm{n}=25)\end{array}$ & $\begin{array}{l}\text { People with } \\
\text { visual } \\
\text { impairments }\end{array}$ & $\begin{array}{l}\text { Students were split into two groups and } \\
\text { watched either a multimedia learning tools } \\
\text { (MMLTs) and read a textbook excerpt, } \\
\text { covering different topics (Acute visual loss } \\
\text { vs cataracts), followed by the other method. }\end{array}$ & $\mathrm{x}$ & & & & & knowledge & $\begin{array}{l}\text { There was no significant difference in student } \\
\text { performance between MMLT and textbook excerpt for } \\
\text { either visual loss or cataract, however the MMLT } \\
\text { cataract group did get higher knowledge scores (mean } \\
75 \% \text { vs. } 61 \%, \mathrm{p}=0.201 \text { ). }\end{array}$ \\
\hline $\begin{array}{l}\text { Dyer et al. } \\
2014^{67}\end{array}$ & $\begin{array}{l}\text { East Midlands, } \\
\text { UK }\end{array}$ & $\begin{array}{l}\text { First and second } \\
\text { year nursing } \\
\text { students }(n=138)\end{array}$ & $\begin{array}{l}\text { People with } \\
\text { disabilities }\end{array}$ & $\begin{array}{l}\text { Students watched a } 30 \text { minute 'Sexual } \\
\text { Respect' DVD to educate on the types of } \\
\text { sexual problems individuals with disabilities } \\
\text { face, tips on initiating the conversation about } \\
\text { sex with patients as well as self-help and } \\
\text { peer support groups that patients can be } \\
\text { directed to. Clinicians and people with } \\
\text { disabilities are featured in the video. }\end{array}$ & $\mathrm{x}$ & $\mathrm{x}$ & & & & $\begin{array}{l}\text { knowledge, } \\
\text { confidence, } \\
\text { attitudes, } \\
\text { competence }\end{array}$ & $\begin{array}{l}\text { Students had significantly greater scores in knowledge, } \\
\text { confidence, comfort, and willingness after watched the } \\
\text { DVD compared to baseline and pre-DVD ( }<00.001) \text {, } \\
\text { with no significant difference between post-DVD and } \\
\text { the } 2 \text { week follow up (except for comfort, which was } \\
\text { statistically significant). Lack of knowledge was cited } \\
\text { as the most common barrier to discussing sex with } \\
\text { patients }(48 \%) \text { but found that the training made it easier } \\
(64 \%) \text {. }\end{array}$ \\
\hline $\begin{array}{l}\text { Provident and } \\
\text { Colmer } 2013 \\
68\end{array}$ & $\begin{array}{l}\text { Camp Kon-o- } \\
\text { Kwee/Spencer in } \\
\text { Pennsylvania, } \\
\text { USA }\end{array}$ & $\begin{array}{l}\text { Level I fieldwork } \\
\text { Occupational } \\
\text { therapy students } \\
(\mathrm{n}=37)\end{array}$ & $\begin{array}{l}\text { Children with } \\
\text { muscular } \\
\text { dystrophy and } \\
\text { related } \\
\text { muscle } \\
\text { diseases }\end{array}$ & $\begin{array}{l}\text { Students participated in a weeklong summer } \\
\text { camp for campers who have muscular } \\
\text { dystrophy or related muscle diseases. } \\
\text { Students interviewed families on medical } \\
\text { care, shadowed nurses, and assisted with } \\
\text { daily routines, transfers, and other counsellor } \\
\text { duties, as necessary. }\end{array}$ & & & $x$ & & & $\begin{array}{l}\text { knowledge, } \\
\text { confidence, } \\
\text { comfort }\end{array}$ & $\begin{array}{l}\text { Students improved their average comfort rating from } \\
4.5 / 10 \text { on day } 1 \text { to } 9.18 \text { on the last day. Students also } \\
\text { qualitatively identified that they improved their skills, } \\
\text { knowledge, relationships, understanding of caregivers } \\
\text { and documentation, and comfort in providing care to } \\
\text { people with muscular dystrophy. }\end{array}$ \\
\hline
\end{tabular}




\begin{tabular}{|c|c|c|c|c|c|c|c|c|c|c|}
\hline $\begin{array}{l}\text { Adib- } \\
\text { Hajbaghery } \\
\text { and Rezaei- } \\
\text { Shahsavarloo } \\
2015^{69}\end{array}$ & $\begin{array}{l}\text { Kashan University } \\
\text { of Medical } \\
\text { Sciences, Iran }\end{array}$ & $\begin{array}{l}\text { Final year nursing } \\
\text { students }(\mathrm{n}=71)\end{array}$ & $\begin{array}{l}\text { Hard of } \\
\text { hearing and } \\
\text { D/deaf people }\end{array}$ & $\begin{array}{l}\text { Nursing students participated in two clinical } \\
\text { simulations with D/deaf and hard of hearing } \\
\text { patients to improve knowledge and } \\
\text { performance and confidence in supporting } \\
\text { hearing impaired individuals. }\end{array}$ & & & & $\mathrm{x}$ & $\begin{array}{l}\text { knowledge, } \\
\text { competence }\end{array}$ & $\begin{array}{l}61.5 \% \text { of students had low-very low levels of } \\
\text { knowledge and } 87.3 \% \text { had weak to very weak } \\
\text { performance in communicating with D/deaf and hard of } \\
\text { hearing patients. There was a significant correlation } \\
\text { between the mean knowledge scores and the students' } \\
\text { performance in the simulation. No students had } \\
\text { knowledge of Persian sign language, but some }(11.3 \%) \\
\text { had a history of caring for a patient with hearing } \\
\text { impairment. }\end{array}$ \\
\hline $\begin{array}{l}\text { VandeWaa et } \\
\text { al. } 2019^{29}\end{array}$ & $\begin{array}{l}\text { University of } \\
\text { South Alabama, } \\
\text { USA }\end{array}$ & $\begin{array}{l}\text { Graduate-level } \\
\text { audiology }(\mathrm{n}=24) \\
\text { undergraduate } \\
\text { nursing }(\mathrm{n}=53) \\
\text { graduate-level } \\
\text { speech language } \\
\text { pathology }(\mathrm{n}=24) \\
\text { students (total } \\
\mathrm{n}=101)\end{array}$ & $\begin{array}{l}\text { Speech or } \\
\text { D/deaf and } \\
\text { hard of } \\
\text { hearing } \\
\text { people }\end{array}$ & $\begin{array}{l}\text { Students completed online training one week } \\
\text { prior to a simulation, which used enhanced } \\
\text { interprofessional education (Sim-IPE). } \\
\text { During the simulation, students worked in } \\
\text { groups of } 3 \text { (one from each field) and } \\
\text { completed } 4 \text { scenarios related to medication } \\
\text { side effects with simulated patients. The } \\
\text { emphasis was on teamwork of the group } \\
\text { with emphasis on effective patient } \\
\text { communication. }\end{array}$ & $\mathrm{x}$ & & & $\mathrm{x}$ & $\begin{array}{l}\text { knowledge, } \\
\text { competence }\end{array}$ & $\begin{array}{l}\text { Students from all } 3 \text { disciplines were found to score } \\
\text { higher in knowledge of medications that impact } \\
\text { communication after the intervention compared to } \\
\text { before. The average pretest score across the disciplines } \\
\text { was } 5.48 \text { ( } \mathrm{SD}=1.53) \text { which significantly }(\mathrm{p}<0.001) \\
\text { increased to } 6.39(\mathrm{SD}=1.29) \text {. Overall students } \\
\text { impressions were positive with improved healthcare } \\
\text { team communication. }\end{array}$ \\
\hline $\begin{array}{l}\text { Tate et al. } \\
2020^{70}\end{array}$ & $\begin{array}{l}\text { The Ohio State } \\
\text { University } \\
\text { College of } \\
\text { Nursing, USA }\end{array}$ & $\begin{array}{l}\text { Second-year } \\
\text { nursing students } \\
(\mathrm{n}=161)\end{array}$ & $\begin{array}{l}\text { People with } \\
\text { communicatio } \\
\mathrm{n} \\
\text { impairments }\end{array}$ & $\begin{array}{l}\text { Nursing students took part in the } \\
\text { StudentSPEACS program which involved a } \\
1 \mathrm{hr} \text {. online training component to improve } \\
\text { communications skills, followed by a } 1.5 \mathrm{hr} \text {. } \\
\text { in class lecture on assessing communication } \\
\text { abilities and strategies, and then a } 1.5 \mathrm{hr} \\
\text { clinical simulation. }\end{array}$ & $\mathrm{x}$ & & & $\mathrm{x}$ & $\begin{array}{l}\text { attitudes, } \\
\text { competency }\end{array}$ & $\begin{array}{l}\text { Students reported improved communication skills after } \\
\text { the training, on average, and } 98 \% \text { of students reported } \\
\text { using StudentSPEACS in the clinical setting. }\end{array}$ \\
\hline $\begin{array}{l}\text { Theoret et al. } \\
2020^{71}\end{array}$ & $\begin{array}{l}\text { Rutgers } \\
\text { University, USA }\end{array}$ & $\begin{array}{l}\text { Medical students } \\
(\mathrm{n}=56,19 \\
\text { members and } 37 \\
\text { non-members })\end{array}$ & $\begin{array}{l}\text { people with } \\
\text { intellectual } \\
\text { and physical } \\
\text { disabilities }\end{array}$ & $\begin{array}{l}\text { Students that are members of the } \\
\text { Involvement with Disability Education and } \\
\text { Advancement (IDEA) partnered with a local } \\
\text { middle/high school for students with } \\
\text { intellectual and physical disabilities. } \\
\text { Students conducted health related workshops } \\
\text { (including sexual education) in the } \\
\text { classroom and did arts, crafts, and exercise } \\
\text { programs to increase independence, } \\
\text { confidence, and self-efficacy. }\end{array}$ & & & $\mathrm{x}$ & & conf & $\begin{array}{l}\text { Regardless of IDEA membership, medical students of } \\
\text { all year perceived themselves to be more comfortable } \\
\text { caring for physically disabled than students with } \\
\text { intellectual disabilities or non-verbal patients after } \\
\text { participating in the program. A higher percentage of } \\
\text { IDEA members answered with a higher comfort level of } \\
5 \text { or greater when indicating comfort with eliciting } \\
\text { information from patients with intellectual disabilities } \\
\text { compared to non-members ( } 89.5 \% \text { vs } 59.5 \% \text {, } \\
\text { p=0.0207). Conversely, non-members had higher } \\
\text { comfort providing care to patients with physical } \\
\text { disabilities than non-members. }\end{array}$ \\
\hline $\begin{array}{l}\text { Watters et al. } \\
2015^{72}\end{array}$ & $\begin{array}{l}\text { New York } \\
\text { University } \\
\text { College of } \\
\text { Dentistry, USA }\end{array}$ & $\begin{array}{l}\text { Fourth year dental } \\
\text { students }(n=364)\end{array}$ & $\begin{array}{l}\text { people with } \\
\text { disabilities }\end{array}$ & $\begin{array}{l}\text { Students participated in instruction before } \\
\text { each clinic session for an hour and a half and } \\
\text { were assigned articles and case studies. } \\
\text { Students participated in a 4-6-week rotation } \\
\text { (half days) in the special needs clinic in } \\
\text { groups of seven to nine students. Students } \\
\text { took turns providing care and serving as } \\
\text { dental assistants to practice skills, improve } \\
\text { comfort, and self-efficacy of students. }\end{array}$ & $\mathrm{x}$ & $\mathrm{x}$ & $\mathrm{x}$ & $\mathrm{x}$ & $\begin{array}{l}\text { competency, } \\
\text { confidence, self- } \\
\text { efficacy, attitudes }\end{array}$ & $\begin{array}{l}\text { Students reported greater confidence in treating people } \\
\text { with intellectual disabilities and medical complexities, } \\
(\mathrm{p}<0.001) \text {. Furthermore, the students agreed less }(2.6 \text {, } \\
\mathrm{SD}=1) \text { than before }(3, \mathrm{SD}=1.1) \text { the rotation that people } \\
\text { with disabilities care less about their oral health } \\
\mathrm{p}<0.001 \text {. People with less previous experience showed } \\
\text { the greatest improvement when dealing with medically } \\
\text { complex patients. }\end{array}$ \\
\hline
\end{tabular}




\begin{tabular}{|c|c|c|c|c|c|c|c|c|c|}
\hline $\begin{array}{l}\text { Vento-Wilson } \\
\text { et al. } 2015^{73}\end{array}$ & $\begin{array}{l}\text { Large Urban } \\
\text { University in } \\
\text { Southern } \\
\text { California, USA }\end{array}$ & $\begin{array}{l}\text { Nursing students } \\
(\mathrm{n}=103)\end{array}$ & $\begin{array}{l}\text { People with } \\
\text { communicatio } \\
\mathrm{n} \\
\text { impairments }\end{array}$ & $\begin{array}{l}\text { Principal investigator conducted a } 1 \mathrm{hr} \text {. } \\
\text { course to train student nurses on } \\
\text { Augmentative and Alternative } \\
\text { communication strategies (AAC) to improve } \\
\text { communication with patients who have } \\
\text { severe communication deficits (SCDs). }\end{array}$ & $\mathrm{x}$ & & & $\begin{array}{l}\text { knowledge, } \\
\text { confidence, } \\
\text { competence }\end{array}$ & $\begin{array}{l}\text { After the AAC training, students felt more confident in } \\
\text { utilizing the tools ( } \mathrm{p}<0.01 \text { ), with } 90 \% \text { feeling some } \\
\text { level of confidence and } 42 \% \text { feeling very confident } \\
\text { compared to } 7 \% \text { before. From the follow-up, } 30 \\
\text { students reported using all } 4 \text { AAC techniques taught. } \\
\text { Students who participated reported that the techniques } \\
\text { were new to them and were helpful in improving } \\
\text { communication with patients with SCDs. }\end{array}$ \\
\hline $\begin{array}{l}\text { Kirshblum et } \\
\text { al. } 2020^{74}\end{array}$ & $\begin{array}{l}\text { Rutgers Medical } \\
\text { School, USA }\end{array}$ & $\begin{array}{l}\text { First-year medical } \\
\text { students }(\mathrm{n}=355)\end{array}$ & $\begin{array}{l}\text { People with } \\
\text { physical } \\
\text { disabilities }\end{array}$ & $\begin{array}{l}\text { The training on caring for people with } \\
\text { physical disabilities was part of the two-year } \\
\text { Health Equity and Social Justice curriculum. } \\
\text { The program discussed prevalence, issues } \\
\text { with accessible health care and health status, } \\
\text { skills to improve care, and perspectives of } \\
\text { people with physical disabilities. The course } \\
\text { included a lecture, video vignette discussion } \\
\text { (2nd year only), and patient panel to } \\
\text { illustrate disparities. }\end{array}$ & $\mathrm{x}$ & $\mathrm{x}$ & & $\begin{array}{l}\text { knowledge, } \\
\text { comfort }\end{array}$ & $\begin{array}{l}\text { Students' knowledge and comfort improved compared } \\
\text { to baseline. For knowledge, there was significantly } \\
\text { increased awareness around different treatment, } \\
\text { homogeneity of the population, health status, } \\
\text { appropriate interaction with service dogs to initiate } \\
\text { encounter, and legal instruments. For comfort, the } \\
\text { initial pre-survey responses were nearly split between } \\
\text { negative and positive }(169 \text { vs } 174) \text {, there was a } 35 \% \\
\text { increase ( } 50.7 \%-85.8 \%) \text { in positive responses between } \\
\text { the pre-to post-surveys ( } p<0.05) \text {. }\end{array}$ \\
\hline \multicolumn{10}{|c|}{ IN SERVICE } \\
\hline $\begin{array}{l}\text { Read and } \\
\text { Rushton } 2013 \\
75\end{array}$ & $\begin{array}{l}\text { University } \\
\text { Hospital of North } \\
\text { Staffordshire, UK }\end{array}$ & $\begin{array}{l}\text { Nurses and other } \\
\text { allied health } \\
\text { professionals ( } \mathrm{n} \\
=157 \text { ) }\end{array}$ & $\begin{array}{l}\text { People with } \\
\text { intellectual } \\
\text { disabilities }\end{array}$ & $\begin{array}{l}\text { A year-long intervention included a series of } \\
8 \text { workshops and a toolkit. The workshops } \\
\text { were led by clinicians and people with ID to } \\
\text { teach about admission, assessment, } \\
\text { discharge planning, and values and attitudes } \\
\text { regarding ID. The toolkit included a resource } \\
\text { directory, assessment tool, DVD, posters, } \\
\text { communication book, cue cards, and leaflets } \\
\text { about good practices to support people with } \\
\text { intellectual disabilities. }\end{array}$ & $\mathrm{x}$ & $\mathrm{x}$ & $\mathrm{x}$ & $\begin{array}{l}\text { knowledge, } \\
\text { competence, } \\
\text { attitudes }\end{array}$ & $\begin{array}{l}\text { Pre- and post-intervention scores show significant } \\
\text { improvement in knowledge, skills, and attitudes (mean } \\
\text { score of } 36.50 \text { pre-intervention increased to } 42.12 \text { post- } \\
\text { intervention; } \mathrm{p}<0.0001) \text { ). }\end{array}$ \\
\hline $\begin{array}{l}\text { Major et al. } \\
2013^{76}\end{array}$ & $\begin{array}{l}33 \text { academic } \\
\text { programs across } \\
\text { the United States }\end{array}$ & $\begin{array}{l}\text { Pediatric and } \\
\text { medicine-pediatric } \\
\text { residents }(\mathrm{n}=114)\end{array}$ & $\begin{array}{l}\text { Autism } \\
\text { spectrum } \\
\text { disorder }\end{array}$ & $\begin{array}{l}\text { A trial piloting } 7 \text { case-based modules on 1) } \\
\text { early warning signs, 2) Screening, 3) } \\
\text { Communication, 4) Diagnosis, 3) Early } \\
\text { Intervention and Education, 6) Treatment, } \\
\text { and 7) Anticipatory Guidance at } 33 \text { different } \\
\text { sites across the US. Each site offered one of } \\
\text { the } 7 \text { modules. }\end{array}$ & $\mathrm{x}$ & & $\mathrm{x}$ & $\begin{array}{l}\text { knowledge, } \\
\text { communication, } \\
\text { confidence, } \\
\text { competence }\end{array}$ & $\begin{array}{l}\text { Participants attitudes improved and knowledge } \\
\text { improved significantly ( } 58.6 \% \text { pre-test to } 75.3 \% \text { post- } \\
\text { intervention, a } 16.7 \% \text { improvement, }<<0.001) \text {. } \\
\text { Participants self-assessment of confidence and } \\
\text { communication also improved significantly (the mean } \\
\text { pre-test score was } 2.26 \text { on a 4-point Likert scale } \\
(1=\text { poor,4=excellent) to a mean of } 3.08 \text { post-test, } \\
\text { p }<0.001)\end{array}$ \\
\hline $\begin{array}{l}\text { Tuffrey- } \\
\text { Wijne et al. } \\
2017^{77}\end{array}$ & $\begin{array}{l}\text { Residential homes } \\
\text { for people with } \\
\text { intellectual } \\
\text { disabilities, UK }\end{array}$ & $\begin{array}{l}\text { Staff in } \\
\text { residential/support } \\
\text { ed living services } \\
(\mathrm{n}=114)\end{array}$ & $\begin{array}{l}\text { People with } \\
\text { intellectual } \\
\text { disabilities }\end{array}$ & $\begin{array}{l}\text { Three-day course consisting of World Café } \\
\text { sessions (w/case studies), presentations, } \\
\text { feedback from people with intellectual } \\
\text { disabilities, and a teaching expert on } \\
\text { discussing death and dying. }\end{array}$ & $\mathrm{x}$ & $\mathrm{x}$ & $\mathrm{x}$ & $\begin{array}{l}\text { competence, } \\
\text { confidence, } \\
\text { communication }\end{array}$ & $\begin{array}{l}\text { Participants valued the opportunity to hear the } \\
\text { perspectives of people with intellectual disabilities and } \\
\text { the World Café, which provided an opportunity to } \\
\text { discuss case studies. Students improved their } \\
\text { knowledge, skills, and confidence on communicating } \\
\text { about death and dying, which was sustained at 3- } \\
\text { months post-intervention. }\end{array}$ \\
\hline $\begin{array}{l}\text { Chiang et al. } \\
2013^{26}\end{array}$ & $\begin{array}{l}\text { Two public } \\
\text { hospitals in } \\
\text { Taiwan }\end{array}$ & $\begin{array}{l}\text { Licensed nursing } \\
\text { staff }(n=62)\end{array}$ & People with & $\begin{array}{l}\text { Nurses watched multi-media interactive } \\
\text { DVD to improve knowledge and assessment } \\
\text { skills. Controls were assigned conventional } \\
\text { classroom education on disability }\end{array}$ & $\mathrm{x}$ & & $\mathrm{x}$ & $\begin{array}{l}\text { knowledge, } \\
\text { competence }\end{array}$ & $\begin{array}{l}\text { Participants had significantly improved assessment } \\
\text { skills and greater knowledge than the experimental } \\
\text { group that was sustained at follow-up assessments } \\
(\mathrm{p}<0.001) \text {. }\end{array}$ \\
\hline
\end{tabular}




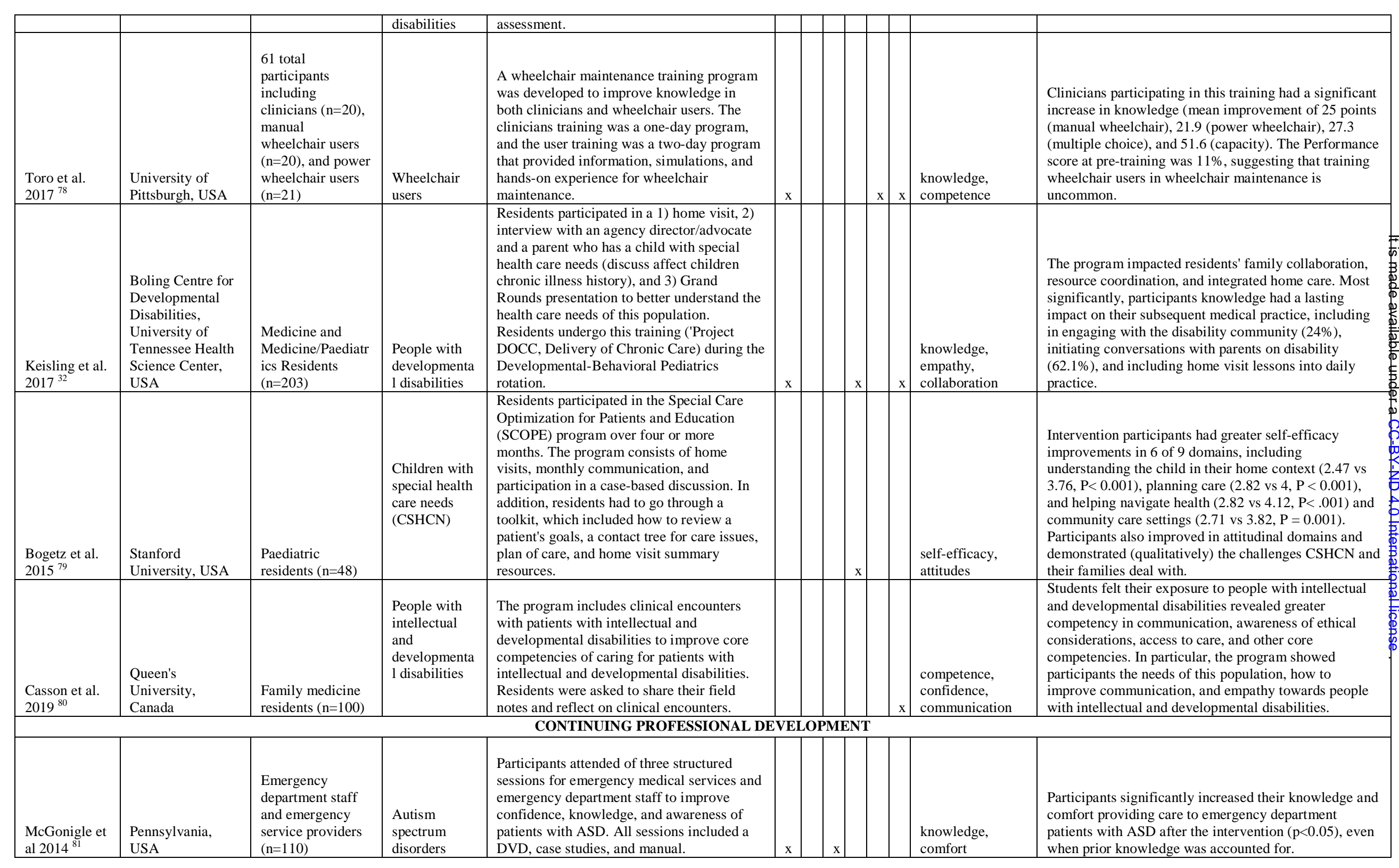




\begin{tabular}{|c|c|c|c|c|c|c|c|c|c|c|c|}
\hline $\begin{array}{l}\text { Doyle and } \\
\text { Bennett } 2014\end{array}$ & $\begin{array}{l}\text { Pacific Northwest, } \\
\text { USA }\end{array}$ & $\begin{array}{l}\text { Occupational } \\
\text { therapists }(n=19)\end{array}$ & $\begin{array}{l}\text { People with } \\
\text { physical } \\
\text { disabilities }\end{array}$ & $\begin{array}{l}\text { An 8-hour workshop for occupational } \\
\text { therapists to improve upper-limb post-stroke } \\
\text { sensory impairments. }\end{array}$ & $\mathrm{x}$ & & & & & $\begin{array}{l}\text { knowledge, } \\
\text { attitudes, } \\
\text { competence }\end{array}$ & $\begin{array}{l}\text { Knowledge, attitudes, client-centered care, and intended } \\
\text { application of skills acquired at the workshop } \\
\text { significantly improved compared to pre-test scores. }\end{array}$ \\
\hline $\begin{array}{l}\text { Dunleavy et } \\
\text { al. } 2018^{83}\end{array}$ & $\begin{array}{l}\text { Two settings: a } \\
\text { central urban } \\
\text { region and a } \\
\text { southern, more } \\
\text { rural part, Rwanda }\end{array}$ & $\begin{array}{l}\text { Physiotherapists } \\
(\mathrm{n}=69)\end{array}$ & $\begin{array}{l}\text { People with } \\
\text { disabilities }\end{array}$ & $\begin{array}{l}\text { Six courses were developed to build clinical } \\
\text { skills, improve decision making and } \\
\text { evaluation, and treatment techniques for } \\
\text { various orthopedic, neurologic, pediatric, } \\
\text { and respiratory conditions, as well as } \\
\text { leadership and advocacy. These were offered } \\
\text { to physiotherapists in over multiple } \\
\text { weekends in the central urban region and a } \\
\text { southern, more rural part. }\end{array}$ & $\mathrm{x}$ & & $\mathrm{x}$ & $\mathrm{x}$ & $\mathrm{x}$ & $\begin{array}{l}\text { quality, } \\
\text { competence }\end{array}$ & $\begin{array}{l}\text { Participants thought they improved the quality of their } \\
\text { physiotherapy care (mean } 6.4 \text { SD } 1.5 \text {, scale of } 0-10 / 10 \\
\text { with } 0 / 10 \text { not at all, } 5 / 10 \text { some, } 10 / 10 \text { great deal), } \\
\text { particularly in treating people with disabilities (mean } \\
7.8 \text { SD } 1.5 \text { ). Overall, their skills improved across the } \\
\text { clinical domains (decision making, treatment, } \\
\text { functional activities, improvement goals, functional } \\
\text { activities, outcome measurement, and adjusting } \\
\text { treatment plans) (average } 8 / 10 \text { for all). }\end{array}$ \\
\hline $\begin{array}{l}\text { Mazurek et } \\
\text { al. } 2020^{24}\end{array}$ & $\begin{array}{l}\text { Various medical } \\
\text { clinics across the } \\
\text { USA }\end{array}$ & $\begin{array}{l}\text { Primary care } \\
\text { providers }(\mathrm{n}=16)\end{array}$ & $\begin{array}{l}\text { Autism } \\
\text { spectrum } \\
\text { disorders }\end{array}$ & $\begin{array}{l}\text { Primary care providers attended } 12 \text { weekly } \\
\text { one-hour video-conference sessions taught } \\
\text { by interdisciplinary experts to improve } \\
\text { knowledge and care for people with ASD. }\end{array}$ & $\mathrm{x}$ & $x$ & $\mathrm{x}$ & & $\mathrm{x}$ & $\begin{array}{l}\text { knowledge, self- } \\
\text { efficacy, } \\
\text { competence }\end{array}$ & $\begin{array}{l}\text { Overall, participants improved self-efficacy in caring } \\
\text { for patients pre-training }(\mathrm{M}=181.9, \mathrm{SD}=54.0) \text { to post- } \\
\text { training }(\mathrm{M}=210.5, \mathrm{SD}=38.9 ; \mathrm{Z}=-2.93, \mathrm{p}=0.003) \\
\text { across all domains. They also improved overall } \\
\text { knowledge (as assessed by the Knowledge Quiz) from } \\
\text { pre-training }(\mathrm{M}=67.3 \%, \mathrm{SD}=11.5 \%) \text { to post-training } \\
(\mathrm{M}=71.9 \%, \mathrm{SD}=12.6 \% ; \mathrm{Z}=-1.79, \mathrm{p}=0.074) \text {. }\end{array}$ \\
\hline $\begin{array}{l}\text { Mazurek et } \\
\text { al. } 2020^{84}\end{array}$ & $\begin{array}{l}10 \text { academic } \\
\text { medical centers in } \\
\text { the USA and } \\
\text { Canada }\end{array}$ & $\begin{array}{l}\text { Primary care } \\
\text { providers who } \\
\text { provide care to } \\
\text { children and to an } \\
\text { underserved } \\
\text { population } \\
(\mathrm{n}=117)\end{array}$ & $\begin{array}{l}\text { Autism } \\
\text { spectrum } \\
\text { disorder }\end{array}$ & $\begin{array}{l}\text { Over } 6 \text { months, } 10 \text { academic medical centers } \\
\text { participated in a randomized control trial of } \\
12 \text { two-hour long training from } \\
\text { interdisciplinary Autism experts through } \\
\text { didactic presentations, PCP-generated case } \\
\text { presentations, and group discussions. }\end{array}$ & $\mathrm{x}$ & & $\mathrm{x}$ & & & $\begin{array}{l}\text { competence, } \\
\text { knowledge, self- } \\
\text { efficacy, barriers } \\
\text { to care }\end{array}$ & $\begin{array}{l}\text { Over } 6 \text { months, there was an increase in autism } \\
\text { screening rates from } 65 \%(95 \% \mathrm{CI}, 40 \%-84 \%) \text { to } 71 \% \\
(95 \% \mathrm{CI}, 52 \%-84 \%)(\mathrm{P}=.63) \text { and a small 6-month } \\
\text { decrease in mean percentage of comorbidities addressed } \\
\text { from } 81 \%(95 \% \mathrm{CI}, 67 \%-90 \%) \text { to } 77 \%(95 \% \mathrm{CI}, 66 \%- \\
86 \%)(\mathrm{P}=.59) \text {. Participants also increased their } \\
\text { knowledge and overall self-efficacy (9\% increase; } 95 \% \\
\mathrm{CI}, 4 \%-13 \% \text {; } \mathrm{P}<.001) \text { and }(29 \% \text { increase; } 95 \% \mathrm{CI} \text {, } \\
25 \%-32 \% ; \mathrm{P}<.001) \text {, respectively, even in follow-up. }\end{array}$ \\
\hline $\begin{array}{l}\text { Locke et al. } \\
2019^{85}\end{array}$ & $\begin{array}{l}\text { University of } \\
\text { Antananarivo, } \\
\text { Madagascar }\end{array}$ & $\begin{array}{l}\text { Doctors } \\
\text { participating in a } \\
\text { rehabilitation } \\
\text { medicine diploma } \\
\text { program }(\mathrm{n}=8) \\
\text { and } \\
\text { physiotherapists } \\
(\mathrm{n}=10)\end{array}$ & $\begin{array}{l}\text { People with } \\
\text { physical } \\
\text { disabilities }\end{array}$ & $\begin{array}{l}\text { A } 400 \text {-hour rehabilitation medicine program } \\
\text { was developed in collaboration with the } \\
\text { University of Leeds and offered in three } \\
\text { sections: general principles of rehabilitation, } \\
\text { neurological condition rehabilitation, and } \\
\text { musculoskeletal condition rehabilitation. } \\
\text { Participants were recruited from different } \\
\text { national and regional hospitals to participate } \\
\text { in the program. }\end{array}$ & $\mathrm{x}$ & & & & & $\begin{array}{l}\text { knowledge, } \\
\text { improved care }\end{array}$ & $\begin{array}{l}\text { Doctors who participated in the program had } \\
\text { significantly improved confidence and competency for } \\
\text { care. Given the limited number of trained rehabilitation } \\
\text { practitioner, the program also increased the number of } \\
\text { trained physicians and physiotherapists in the country in } \\
\text { a sustainable way. }\end{array}$ \\
\hline $\begin{array}{l}\text { Edinger et al. } \\
2019^{86}\end{array}$ & $\begin{array}{l}\text { Rutgers } \\
\text { University Office } \\
\text { of Continuing } \\
\text { Professional } \\
\text { Education, USA }\end{array}$ & $\begin{array}{l}\text { First responders } \\
(\mathrm{n}=33)\end{array}$ & $\begin{array}{l}\text { People with } \\
\text { developmenta } \\
\text { l disabilities }\end{array}$ & $\begin{array}{l}\text { A one-hour online workshop was held to } \\
\text { improve disaster and critical care responders } \\
\text { (knowledge and self-efficacy for working } \\
\text { with people with developmental disabilities. } \\
\text { The intervention included an overview of } \\
\text { developmental disabilities, training on } \\
\text { communication, and identification and } \\
\text { interaction techniques. }\end{array}$ & $\mathrm{x}$ & & & & & $\begin{array}{l}\text { knowledge, self- } \\
\text { efficacy, }\end{array}$ & $\begin{array}{l}\text { Overall knowledge improved from } 66 \% \text { correct to } 81 \% \\
\text { correct, including significant }(\mathrm{p}<0.05) \text { changes in } 4 \\
\text { domains. Self-efficacy also improved from } 53 \% \text { on the } \\
\text { pretest to } 92 \% \text { on the posttest, indicating an increase of } \\
39 \% \text { with significant }(\mathrm{p}<0.05) \text { changes in all } 10 \\
\text { domains. }\end{array}$ \\
\hline $\begin{array}{l}\text { Auberry et al. } \\
2019^{87}\end{array}$ & $\begin{array}{l}\text { Indiana University } \\
\text { School of } \\
\text { Nursing, USA }\end{array}$ & $\begin{array}{l}\text { Volunteer direct } \\
\text { support } \\
\text { professionals that } \\
\text { worked with } \\
\end{array}$ & People with & $\begin{array}{l}\text { Volunteers participated in sessions on } \\
\text { medication administration for people with } \\
\text { intellectual and developmental disabilities. } \\
\text { The session was facilitated by a doctoral- }\end{array}$ & $\mathrm{x}$ & & & $\mathrm{x}$ & & $\begin{array}{l}\text { knowledge, } \\
\text { confidence }\end{array}$ & $\begin{array}{l}\text { Students significantly improved their knowledge and } \\
\text { skills to administer a variety of medications through } \\
\text { participating in the program ( }<0.05 \text { for all topics). } \\
\text { They expressed greater confidence in treating patients }\end{array}$ \\
\hline
\end{tabular}




\begin{tabular}{|c|c|c|c|c|c|c|c|c|c|c|}
\hline & & $\begin{array}{l}\text { intellectual and } \\
\text { developmental } \\
\text { disabilities }(n=12)\end{array}$ & $\begin{array}{l}\text { intellectual } \\
\text { and } \\
\text { developmenta } \\
1 \text { disabilities }\end{array}$ & $\begin{array}{l}\text { trained and bachelor-trained registered nurse } \\
(\mathrm{RN}) \text { certified in developmental disability } \\
\text { nursing covered various medication practice } \\
\text { instructions through didactic lectures and } \\
\text { simulations. }\end{array}$ & & & & & & $\begin{array}{l}\text { ( } \mathrm{p}<0.02 \text { for all metrics) and appreciated the opportunity } \\
\text { to practice skills. }\end{array}$ \\
\hline $\begin{array}{l}\text { Olowoyeye et } \\
\text { al. } 2019^{88}\end{array}$ & $\begin{array}{l}\text { Ifo Local } \\
\text { Government Area, } \\
\text { Ogun State, } \\
\text { Nigeria }\end{array}$ & $\begin{array}{l}\text { Maternal and } \\
\text { child health care } \\
\text { workers } \\
\text { (MCHWs }(\mathrm{n}=65)\end{array}$ & $\begin{array}{l}\text { Children with } \\
\text { visual } \\
\text { impairments }\end{array}$ & $\begin{array}{l}\text { MCHWs participated in a pre-test and focus } \\
\text { group discussion on leading causes, } \\
\text { identification, management, and prevention } \\
\text { of childhood blindness. Participants then } \\
\text { participated in a lecture and practical session } \\
\text { on eye diseases and asked to log any } \\
\text { referrals following the intervention. After } 3 \\
\text { months, the participants did a post-test } \\
\text { questionnaire. }\end{array}$ & $\mathrm{x}$ & & & $\mathrm{x}$ & knowledge & $\begin{array}{l}\text { Participant's knowledge scores increased significantly } \\
\text { after the training, particularly in identifying common } \\
\text { diseases, locally available vitamins, and conjunctivitis } \\
\text { prevention. } 45.9 \% \text { MCHWs felt they had inadequate } \\
\text { training prior but did not significantly increase } \\
\text { knowledge in immunizations for preventable blindness, } \\
\text { or glaucoma/cataract treatment. Six children were } \\
\text { referred for services because of the program. }\end{array}$ \\
\hline $\begin{array}{l}\text { Cameron et } \\
\text { al. } 2017^{89}\end{array}$ & $\begin{array}{l}\text { Metropolitan } \\
\text { Hospital in } \\
\text { Australia }\end{array}$ & $\begin{array}{l}\text { Heath } \\
\text { professionals } \\
(\mathrm{n}=52)\end{array}$ & $\begin{array}{l}\text { People with } \\
\text { Aphasia }\end{array}$ & $\begin{array}{l}\text { The intervention programme consisted of a } \\
\text { 1-h lecture that discussed strategies for } \\
\text { communicating effectively with people with } \\
\text { aphasia (PWA). This lecture was provided } \\
\text { by a speech language pathologist member of } \\
\text { the research team. This was immediately } \\
\text { followed by a 15-20-minute practical } \\
\text { conversation with a PWA to facilitate } \\
\text { application of the discussed strategies and } \\
\text { approaches. }\end{array}$ & $\mathrm{x}$ & & $\mathrm{x}$ & & $\begin{array}{l}\text { confidence, } \\
\text { knowledge }\end{array}$ & $\begin{array}{l}\text { The participants self-reported a mean confidence rating } \\
\text { of } 75.8 \mathrm{~mm}(\mathrm{SD}=12.2) \text { after completing the training } \\
\text { which was significantly higher than the rating of } 46.6 \\
\mathrm{~mm}(\mathrm{SD}=15.7) \text { before completing the training }(\mathrm{p}< \\
.001) \text {. At the pre-intervention assessment, participants } \\
\text { identified a median of three strategies for facilitating } \\
\text { communication with PWA. Following the training, the } \\
\text { HPs were able to identify a total of } 35 \text { strategies. }\end{array}$ \\
\hline $\begin{array}{l}\text { Mac Giolla } \\
\text { Phadraig et } \\
\text { al. } 2015^{37}\end{array}$ & $\begin{array}{l}\text { Residential care } \\
\text { homes in Dublin, } \\
\text { Ireland }\end{array}$ & $\begin{array}{l}\text { key care staff of } \\
\text { residential home } \\
(n=22) \text {, people } \\
\text { with ID }(n=155) \\
\end{array}$ & $\begin{array}{l}\text { People with } \\
\text { intellectual } \\
\text { disabilities }\end{array}$ & $\begin{array}{l}22 \text { trainees from intervention group attended } \\
\text { a multi-tiered oral health educational } \\
\text { program on the oral health and oral hygiene } \\
\text { of people with intellectual disabilities. } \\
\text { Trainees were expected to train peers. A } \\
\text { control group lived in centers where staff } \\
\text { received no training. }\end{array}$ & $\mathrm{x}$ & $\mathrm{x}$ & & & $\begin{array}{l}\text { knowledge, } \\
\text { attitude, self- } \\
\text { efficacy }\end{array}$ & $\begin{array}{l}\text { The intervention improved the oral health-related } \\
\text { knowledge, attitude, self-efficacy, and behavior of } \\
\text { residential care-staff who provided care to a population } \\
\text { of people with ID. A reduction in gingivitis and plaque } \\
\text { scores were found in the people who lived in care } \\
\text { homes where staff participated by } 10.5 \% \text { and } 8.5 \% \\
\text { respectively, though not statistically significant between } \\
\text { groups. }\end{array}$ \\
\hline $\begin{array}{l}\text { Dagnan et al. } \\
2018^{17}\end{array}$ & $\begin{array}{l}\text { An English } \\
\text { Improving Access } \\
\text { to Psychological } \\
\text { Therapies (IAPT) } \\
\text { service, UK }\end{array}$ & Therapists $(n=68)$ & $\begin{array}{l}\text { People with } \\
\text { intellectual } \\
\text { disabilities }\end{array}$ & $\begin{array}{l}\text { Therapists working in an Improving Access } \\
\text { to Psychological Therapies Service } \\
\text { participated in a one- or two-day workshop } \\
\text { on supporting people with intellectual. The } \\
\text { workshop was divided into } 8 \text { sessions, } \\
\text { including introduction to intellectual } \\
\text { disability, stigma, assessment, adaptation of } \\
\text { therapeutic techniques, approaches, and local } \\
\text { services/resources. }\end{array}$ & $\mathrm{x}$ & & & & $\begin{array}{l}\text { confidence, self- } \\
\text { efficacy, attitude }\end{array}$ & $\begin{array}{l}\text { Therapists had improved confidence, self-efficacy, and } \\
\text { attitudes across both therapist groups. There was a } \\
\text { statistically significant improvement from pre- to post- } \\
\text { training ( }<0.001 \text { ), and while improvements were } \\
\text { sustained 3-months later, there was not a statistical } \\
\text { significance. Qualitatively, several respondents noted } \\
\text { the workshop increased awareness and sensitivity, } \\
\text { adapted materials, communications, and interventions. }\end{array}$ \\
\hline $\begin{array}{l}\text { Catteau et al. } \\
2013^{90}\end{array}$ & $\begin{array}{l}\text { Dental practices } \\
\text { across France }\end{array}$ & Dentists $(n=26)$ & $\begin{array}{l}\text { People with } \\
\text { disabilities }\end{array}$ & $\begin{array}{l}\text { A 10-module online curriculum 'Autonomy } \\
\text { and Oral Health' (30-50h of coursework) } \\
\text { covered dental health for persons with } \\
\text { disabilities, ranging from lack of autonomy } \\
\text { to special care dentistry. Dentists also } \\
\text { conducted an oral health promotion course } \\
\text { for people with disabilities at institutions for } \\
\text { children and adults. }\end{array}$ & $\mathrm{x}$ & $\mathrm{x}$ & & $\mathrm{x}$ & $\begin{array}{l}\text { knowledge, self- } \\
\text { efficacy }\end{array}$ & $\begin{array}{l}\text { Dentists scored their self-efficacy significantly higher } \\
\text { both after the online training }(\mathrm{p}<0.01) \text { and after the } \\
\text { course }(\mathrm{p}<0.05) \text {. Overall, } 94 \% \text { of the staff/caregivers of } \\
\text { the institutions where dentists implemented their } \\
\text { interventions indicated an improvement in the oral } \\
\text { hygiene of the residents. }\end{array}$ \\
\hline
\end{tabular}




\begin{tabular}{|c|c|c|c|c|c|c|c|c|c|c|c|c|}
\hline $\begin{array}{l}\text { Clark et al. } \\
2019^{33}\end{array}$ & $\begin{array}{l}\text { University of } \\
\text { Rwanda }\end{array}$ & $\begin{array}{l}\text { Physical } \\
\text { Therapists }(\mathrm{n}=66)\end{array}$ & $\begin{array}{l}\text { Children with } \\
\text { physical } \\
\text { disabilities }\end{array}$ & $\begin{array}{l}\text { Physical therapists, to improve paediatric } \\
\text { clinical decision making, were taught for a } \\
\text { total of } 48 \text { hours over the course of } 4 \\
\text { weekends. Teaching involved classroom } \\
\text { lectures, small group case studies, laboratory } \\
\text { skills practice, and clinical visits to } \\
\text { participants place of work. }\end{array}$ & $\mathrm{x}$ & $\mathrm{x}$ & & $\mathrm{x}$ & $\mathrm{x}$ & $\begin{array}{l}\text { knowledge, } \\
\text { confidence, } \\
\text { competence }\end{array}$ & $\begin{array}{l}\text { Physical therapists' performance on paediatric } \\
\text { rehabilitation knowledge questionnaire improved from } \\
\text { an average of } 53.7 \% \text { pre course to } 82.2 \% \text { after the } \\
\text { course. Participants indicated an increased confidence } \\
\text { in the eight clinical skills taught, which was also } \\
\text { observed by US based therapists on clinic visits. }\end{array}$ & \\
\hline $\begin{array}{l}\text { Quinn and } \\
\text { Smolinski } \\
2018^{91}\end{array}$ & $\begin{array}{l}\text { National } \\
\text { Association of } \\
\text { School Nurses } \\
\text { annual } \\
\text { conference, } \\
\text { USAghent }\end{array}$ & $\begin{array}{l}\text { School nurses } \\
(\mathrm{n}=248)\end{array}$ & $\begin{array}{l}\text { People with } \\
\text { intellectual } \\
\text { disabilities }\end{array}$ & $\begin{array}{l}\text { School nurses participated in three-hour } \\
\text { educational sessions that focused on } \\
\text { increasing their knowledge related to pain } \\
\text { and pain assessment for students with } \\
\text { intellectual disabilities. }\end{array}$ & $\mathrm{x}$ & & & & & $\begin{array}{l}\text { knowledge, } \\
\text { competence }\end{array}$ & $\begin{array}{l}\text { Before, school nurses rating their difficulty in } \\
\text { identifying pain in ID students ranked it a } 6 \text { (range } 1 \text { - } \\
10, \mathrm{SD}=1.9 \text { ) compared to } 3.41 \text { in neurotypical (range } 0 \text { - } \\
10, \mathrm{SD}=2.25 \text { ) children. This decreased to } 2 \text { (range 1-7, } \\
\mathrm{SD}=1.8, \mathrm{p}<0.0001 \text { ) post-session. } 74 \% \text { of nurses } \\
\text { changed their practices and reported significant } \\
\text { decreases in difficulty when assessing pain ( }<<0.0006- \\
0.1 \text { ) six months after the intervention. }\end{array}$ & \\
\hline $\begin{array}{l}\text { Phlypo et al. } \\
2018^{92}\end{array}$ & $\begin{array}{l}\text { Ghent University } \\
\text { and a local } \\
\text { residential facility } \\
\text { in Belgium }\end{array}$ & $\begin{array}{l}\text { Residential } \\
\text { facility caregivers } \\
(\mathrm{n}=99, \text { control } \\
=42, \\
\text { intervention=37) }\end{array}$ & $\begin{array}{l}\text { People with } \\
\text { intellectual } \\
\text { disabilities }\end{array}$ & $\begin{array}{l}\text { Students initiated an oral health promotion } \\
\text { project for people with intellectual } \\
\text { disabilities and their caregivers living in a } \\
\text { residential care home. Facility caregivers } \\
\text { were randomized and those in the } \\
\text { intervention group received a booklet } \\
\text { including chapters on oral hygiene, nutrition, } \\
\text { visiting the dentist and practical tips on } \\
\text { providing health care for people with } \\
\text { disabilities and an information session for } \\
\text { residents and caregivers. }\end{array}$ & $\mathrm{x}$ & & & & & $\begin{array}{l}\text { knowledge, } \\
\text { attitudes, self- } \\
\text { efficacy }\end{array}$ & $\begin{array}{l}\text { Caregivers significantly improved their knowledge } \\
\text { index in the intervention group (mean of } 5.9 \text { pre- } \\
\text { intervention to } 7.1 \text { post-intervention, p=0.03). Both } \\
\text { groups improved their behaviors, attitudes, and self- } \\
\text { efficacy, but not in the intervention group and not } \\
\text { significantly in the control group. }\end{array}$ & 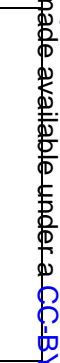 \\
\hline $\begin{array}{l}\text { Sanchez et al. } \\
2017^{93}\end{array}$ & $\begin{array}{l}\text { Federally } \\
\text { Qualified Health } \\
\text { Care Centre in } \\
\text { Nogales, Arizona, } \\
\text { USA }\end{array}$ & $\begin{array}{l}\text { Female } \\
\text { community } \\
\text { health-care } \\
\text { workers }(\mathrm{n}=12), 4 \\
\text { of which } \\
\text { participated in } \\
\text { facilitator training }\end{array}$ & $\begin{array}{l}\text { Hard of } \\
\text { hearing and } \\
\text { D/deaf people }\end{array}$ & $\begin{array}{l}\text { CHWs attended two-hour focus groups and a } \\
\text { 3-hour workshop that included didactic } \\
\text { methods and simulation. CHWs who } \\
\text { participated in the workshop held } \\
\text { community screenings and some participated } \\
\text { in an additional } 8 \text { sessions over } 6 \text { weeks to } \\
\text { become facilitators. The goal was to improve } \\
\text { screening, understanding, referrals and } \\
\text { effective communication. }\end{array}$ & $\mathrm{x}$ & $\mathrm{x}$ & $\mathrm{x}$ & $\mathrm{x}$ & & $\begin{array}{l}\text { knowledge, } \\
\text { confidence, } \\
\text { competence }\end{array}$ & $\begin{array}{l}\text { CHWs knowledge and confidence regarding peer } \\
\text { support for community members with hearing loss } \\
\text { increased and they were able to apply their skills to } \\
\text { specific situations and help address the audiological } \\
\text { needs of the community. }\end{array}$ & 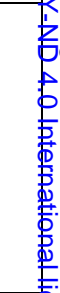 \\
\hline $\begin{array}{l}\text { Mac Giolla } \\
\text { Phadraig et } \\
\text { al. } 2012^{38}\end{array}$ & $\begin{array}{l}\text { Community-based } \\
\text { residential care } \\
\text { homes in Dublin, } \\
\text { Ireland }\end{array}$ & $\begin{array}{l}\text { Residential care } \\
\text { home staff } \\
(\mathrm{n}=219)\end{array}$ & $\begin{array}{l}\text { People with } \\
\text { intellectual } \\
\text { disabilities }\end{array}$ & $\begin{array}{l}22 \text { trainees from intervention group attended } \\
\text { a day long training session provided by a } \\
\text { team of oral health trainers (lectures, group } \\
\text { based practical sessions, role play, group } \\
\text { feedback). Trainees were given training } \\
\text { packs to go back to care home and train } \\
\text { peers. The control group received no } \\
\text { training. The goal was to see if training can } \\
\text { improve the Knowledge, Attitudes, Self- } \\
\text { efficacy, and Reported Behaviour (KBAS) } \\
\text { of the intellectual disability staff. }\end{array}$ & $\mathrm{x}$ & & $x$ & $x$ & & $\begin{array}{l}\text { knowledge, } \\
\text { competence, } \\
\text { attitudes }\end{array}$ & $\begin{array}{l}\text { The mean knowledge index score for the intervention } \\
\text { group rose from } \mathrm{K}=7.23(\mathrm{SD}=1.34 \text { ) to } \mathrm{K}=7.86 \\
(\mathrm{SD}=1.27) \mathrm{p}<0.0001 . \mathrm{BAS} \text { scores also significantly } \\
\text { increased from } 4.73 \text { ( } \mathrm{SD}=1.32 \text { ) to } 5.42(\mathrm{SD}=1.51 \text { ), } \\
\text { p }<0.0001 \text {. There was no significant difference in } \mathrm{K} \text { and } \\
\text { BAS scores in the control group. }\end{array}$ & d \\
\hline $\begin{array}{l}\text { Pasco et al. } \\
2014^{36}\end{array}$ & $\begin{array}{l}\text { Cities across } \\
\text { Romania } \\
\text { (Bucharest, Cluj, } \\
\text { Iasi, Timisoara) } \\
\end{array}$ & $\begin{array}{l}\text { Professional } \\
\text { training } \\
\text { programme } \\
(\mathrm{n}=1075) \text { online }\end{array}$ & & $\begin{array}{l}\text { Three-stage training program to increase } \\
\text { knowledge of ASD and share best practices } \\
\text { (two 3-day courses, six 6-day courses, and } \\
\text { 30h of supervised practice). Two online }\end{array}$ & $\mathrm{x}$ & & $\mathrm{x}$ & & $\mathrm{x}$ & $\begin{array}{l}\text { knowledge, } \\
\text { competence }\end{array}$ & $\begin{array}{l}\text { Scores on the ASD knowledge test were found to } \\
\text { increase from } 6.9 \text { before to } 11 \text { after the training session } \\
(\mathrm{p}<0.05) \text {. Six-months after, } 94 \% \text { reported using the } \\
\text { knowledge and skills in current practice. Overall, the }\end{array}$ & \\
\hline
\end{tabular}




\begin{tabular}{|c|c|c|c|c|c|c|c|c|c|}
\hline & & $\begin{array}{l}\text { course }(\mathrm{n}=613 \\
\text { general } \\
\text { practitioners } \\
\text { (GPs) snd } \\
\text { psychiatrists) }\end{array}$ & $\begin{array}{l}\text { Autism } \\
\text { spectrum } \\
\text { disorder }\end{array}$ & $\begin{array}{l}\text { courses for GPs and Psychiatrists on } \\
\text { improving diagnosis and treatment were also } \\
\text { offered, among other initiatives as part of a } \\
\text { nationwide project to improve awareness and } \\
\text { social inclusion of people with ASD. }\end{array}$ & & & & & $\begin{array}{l}\text { program enhanced knowledge and practical experience } \\
\text { among medical professionals and other specialists } \\
\text { working with children and young people with ASD. }\end{array}$ \\
\hline $\begin{array}{l}\text { Figueiras et } \\
\text { al. } 2014^{94}\end{array}$ & $\begin{array}{l}18 \text { Municipal } \\
\text { Health Units in } \\
\text { Belém, Pará, } \\
\text { Brazil }\end{array}$ & $\begin{array}{l}\text { Health } \\
\text { professionals in } \\
\text { primary care } \\
(\mathrm{n}=221)\end{array}$ & $\begin{array}{l}\text { Cerebral } \\
\text { palsy, autism, } \\
\text { and preterm } \\
\text { newborns }\end{array}$ & $\begin{array}{l}\text { A course including lectures, discussion, } \\
\text { videos, readings, and practical assessments } \\
\text { related to assessing developmental } \\
\text { disabilities. Participants were followed up 1- } \\
3 \text { years later to see the impact on their } \\
\text { practice. }\end{array}$ & & $\mathrm{x}$ & $\mathrm{x}$ & $\begin{array}{l}\text { knowledge, } \\
\text { competence }\end{array}$ & $\begin{array}{l}\text { Health practitioners who participated significantly } \\
\text { increased their knowledge and skills of child } \\
\text { development }(\mathrm{p}<0.001) \text {. Mother's also identified that } \\
\text { practitioners asked more about child development and } \\
\text { intervened more after participating. }\end{array}$ \\
\hline
\end{tabular}


medRxiv preprint doi: https://doi.org/10.1101/2021.08.03.21261522; this version posted August 4, 2021. The copyright holder for this preprint (which was not certified by peer review) is the author/funder, who has granted medRxiv a license to display the preprint in perpetuity.

It is made available under a CC-BY-ND 4.0 International license .

\section{REFERENCES}

1. World Bank and World Health Organization. World Report on Disability. 2011:350. https://www.who.int/disabilities/world_report/2011/report.pdf?ua=1

2. $\quad$ Kuper H, Heydt P. The Missing Billion: Access to Health Services for 1 Billion People with Disabilities. 2019:28. https://www.Ishtm.ac.uk/TheMissingBillion

3. Convention on the Rights of Persons with Disabilities (CRPD), (2006). Accessed 2020/07/11/20:53:45. https://www.un.org/development/desa/disabilities/convention-on-therights-of-persons-with-disabilities.html

4. A new landmark resolution on disability adopted at the 74th World Health Assembly. World Health Organization; 2021. https://www.who.int/news/item/27-05-2021-a-new-landmarkresolution-on-disability-adopted-at-the-74th-world-health-assembly

5. Shakespeare T, Kleine I. Educating Health Professionals about Disability: A Review of Interventions. Health and Social Care Education. 2013;2(2):20-37. doi:10.11120/hsce.2013.00026

6. Ioerger M, Flanders RM, French-Lawyer JR, Turk MA. Interventions to Teach Medical Students About Disability: A Systematic Search and Review. Am J Phys Med Rehabil. Jul 2019;98(7):577-599. doi:10.1097/PHM.0000000000001154

7. Ceglio K, Rispoli MJ, Flake EM. Training Medical Professionals to Work with Patients with Neurodevelopmental Disorders: A Systematic Review. Developmental Neurorehabilitation. 2020 Oct 2;23(7):463-73.

8. Guaranteeing Medicare - Improving the health of people with intellectual disability. 2021. https://www.health.gov.au/sites/default/files/documents/2021/05/guaranteeing-medicareimproving-the-health-of-people-with-intellectual-disability.pdf

9. Health Education E. The Oliver McGowan Mandatory Training in Learning Disability and Autism. Health Education England. 2020/08/07/T15:20:04+01:00 2020;

10. World Health O. Classifying health workers: Mapping occupations to the international standard classification. 2010. 2010/04/29/. Accessed 2021/06/16/.

https://www.who.int/hrh/statistics/Health workers classification.pdf

11. Ouzzani M, Hammady H, Fedorowicz Z, Elmagarmid A. Rayyan - a web and mobile app for systematic reviews. Systematic Reviews. 2016 2016;5(1)doi:10.1186/s13643-016-0384-4

12. Page MJ, McKenzie JE, Bossuyt PM, et al. The PRISMA 2020 statement: an updated guideline for reporting systematic reviews. BMJ. 2021/03/29/ 2021;372:n71.

doi:10.1136/bmj.n71

13. Bright T, Wallace S, Kuper H. A Systematic Review of Access to Rehabilitation for People with Disabilities in Low- and Middle-Income Countries. Int J Environ Res Public Health. 2018;15(10)doi:10.3390/ijerph15102165

14. Gilmore M, Sturgeon A, Thomson C, et al. Changing medical students' attitudes to and knowledge of deafness: a mixed methods study. Bmc Medical Education. Jun 2019;19227. doi:10.1186/s12909-019-1666-Z

15. Bank TW. World Bank Country and Lending Groups. World Bank Group. Accessed 16 June, 2021. 
medRxiv preprint doi: https://doi.org/10.1101/2021.08.03.21261522; this version posted August 4, 2021. The copyright holder for this preprint (which was not certified by peer review) is the author/funder, who has granted medRxiv a license to display the preprint in perpetuity. It is made available under a CC-BY-ND 4.0 International license .

16. Bailey N, Kaarto P, Burkey J, Bright D, Sohn M. Evaluation of an American Sign Language co-curricular training for pharmacy students. Currents in Pharmacy Teaching and Learning. Jan 2021;13(1):68-72. doi:10.1016/j.cptl.2020.08.002

17. Dagnan D, Masson J, Thwaites R, James A, Hatton C. Training therapists to work with people with intellectual disability in Improving Access to Psychological Therapies (IAPT) services. Journal of applied research in intellectual disabilities : JARID. 2018;31(5):760-767. doi:http://dx.doi.org/10.1111/jar.12427

18. Salama FS, Al-Balkhi BK. Effectiveness of educational intervention of oral health for special needs on knowledge of dental students in Saudi Arabia. Disability and Health Journal. Jan 2020;13(1)100789. doi:10.1016/j.dhjo.2019.03.005

19. Steedman M, Abouammoh M, Sharma S. Multimedia learning tools for teaching undergraduate ophthalmology: Results of a randomized clinical study. Canadian Journal of Ophthalmology. 2012;47(1):66-71. doi:http://dx.doi.org/10.1016/j.jcjo.2011.12.006

20. Abdi R, Metcalf E. Exploring attitudes of medical students towards intellectual disabilities. Advances in Mental Health and Intellectual Disabilities. Aug 2020;14(5):125-136. doi:10.1108/amhid-01-2020-0002

21. Lynch J, Last J, Dodd P, Stancila D, Linehan C. 'Understanding Disability': Evaluating a contact-based approach to enhancing attitudes and disability literacy of medical students. Disability and Health Journal. Jan 2019;12(1):65-71. doi:10.1016/j.dhjo.2018.07.007

22. Iannuzzi D, Rissmiller P, Duty SM, Feeney S, Sullivan M, Curtin C. Addressing a Gap in Healthcare Access for Transition-Age Youth with Autism: A Pilot Educational Intervention for Family Nurse Practitioner Students. Journal of Autism and Developmental Disorders. Apr 2019;49(4):1493-1504. doi:10.1007/s10803-018-3846-9

23. Cheek C, Hays R, Smith J, Allen P. Improving case study research in medical education: a systematised review. Medical Education. 2018/05// 2018;52(5):480-487.

doi:10.1111/medu.13469

24. Mazurek MO, Stobbe G, Loftin R, et al. ECHO Autism Transition: Enhancing healthcare for adolescents and young adults with autism spectrum disorder. Autism. Apr 2020;24(3):633644. 1362361319879616. doi:10.1177/1362361319879616

25. Sohl K, Mazurek MO, Brown R. ECHO Autism: Using Technology and Mentorship to Bridge Gaps, Increase Access to Care, and Bring Best Practice Autism Care to Primary Care. Clinical Pediatrics. 2017;56(6):509-511. doi:http://dx.doi.org/10.1177/0009922817691825

26. Chiang HC, Lin FY, Hwu YJ. Disability Assessment: The Efficacy of Multimedia Interactive Nurse Education. Journal of Nursing Research. Jun 2013;21(2):83-93. doi:10.1097/jnr.0b013e3182921f5a

27. Watmough S, Leftwick P, Alexander-White S. An evaluation of medical students' views on the introduction of a community placement and its impact on their understanding of patients with disabilities. Education for primary care : an official publication of the Association of Course Organisers, National Association of GP Tutors, World Organisation of Family Doctors. 2014;25(1):36-42.

28. Hensel D, Malinowski C, Watts PA. Implementing a Pediatric Camp Clinical for PreLicensure Education. Nursing education perspectives. 2015;36(1):60-61. doi:https://dx.doi.org/10.5480/12-871.1

29. VandeWaa E, Bealle Rudd A, Estis JM, Gordon-Hickey S. Safe Medication Administration in Patients with Communication Disorders: A Simulation-Enhanced Interprofessional Education Approach. Journal of allied health. 2019;48(4):257-262. 
medRxiv preprint doi: https://doi.org/10.1101/2021.08.03.21261522; this version posted August 4, 2021. The copyright holder for this preprint (which was not certified by peer review) is the author/funder, who has granted medRxiv a license to display the preprint in perpetuity. It is made available under a CC-BY-ND 4.0 International license .

30. Sheepway L, Lincoln M, McAllister S. Impact of placement type on the development of clinical competency in speech-language pathology students. International journal of language \& communication disorders / Royal College of Speech \& Language Therapists. 2014;49(2):189203. doi:http://dx.doi.org/10.1111/1460-6984.12059

31. Saldert C, Forsgren E, Hartelius L. Teaching medical students about communication in speech-language disorders: Effects of a lecture and a workshop. International Journal of SpeechLanguage Pathology. 2016;18(6):571-579. doi:10.3109/17549507.2016.1143975

32. Keisling BL, Bishop EA, Kube DA, Roth JM, Palmer FB. Long-term pediatrician outcomes of a parent led curriculum in developmental disabilities. Research in Developmental Disabilities. 2017;60:16-23. doi:http://dx.doi.org/10.1016/j.ridd.2016.11.004

33. Clark K, Smith CNW, Kohls L, et al. A global health training model for teaching pediatric clinical decision making skills to Rwandan physical therapists: A case report. Physiotherapy Theory and Practice. Sep 2019;35(9):891-903. doi:10.1080/09593985.2018.1458263

34. Symons AB, Morley CP, McGuigan D, Akl EA. A curriculum on care for people with disabilities: Effects on medical student self-reported attitudes and comfort level. Disability and Health Journal. Jan 2014;7(1):88-95. doi:10.1016/j.dhjo.2013.08.006

35. Garavatti E, Tucker J, Pabian PS. Utilization of an Interprofessional Integrated Clinical Education Experience to Improve Medical and Physical Therapy Student Comfort in Treating Patients with Disabilities. Education for Health. Sep-Dec 2018;31(3):155-162. doi:10.4103/efh.EfH_177_17

36. Pasco G, Clark B, Dragan I, et al. A training and development project to improve services and opportunities for social inclusion for children and young people with autism in Romania. Autism. Oct 2014;18(7):827-831. doi:10.1177/1362361314524642

37. Mac Giolla Phadraig C, Guerin S, Nunn J. Should we educate care staff to improve the oral health and oral hygiene of people with intellectual disability in residential care? Real world lessons from a randomized controlled trial. Special care in dentistry : official publication of the American Association of Hospital Dentists, the Academy of Dentistry for the Handicapped, and the American Society for Geriatric Dentistry. 2015;35(3):92-98.

doi:http://dx.doi.org/10.1111/scd.12102

38. Mac Giolla Phadraig C, Guerin S, Nunn J. Train the trainer? A randomized controlled trial of a multi-tiered oral health education programme in community-based residential services for adults with intellectual disability. Community dentistry and oral epidemiology.

2013;41(2):182-92. doi:https://dx.doi.org/10.1111/cdoe.12006

39. Thew D, Smith SR, Chang C, Starr M. The Deaf Strong Hospital Program: A Model of Diversity and Inclusion Training for First-Year Medical Students. Academic Medicine. Nov 2012;87(11):1496-1500. doi:10.1097/ACM.0b013e31826d322d

40. Smith P, Ooms A, Marks-Maran D. Active involvement of learning disabilities service users in the development and delivery of a teaching session to pre-registration nurses: Students' perspectives. Nurse education in practice. 2016;16(1):111-118.

doi:http://dx.doi.org/10.1016/j.nepr.2015.09.010

41. Lapinski J, Colonna C, Sexton P, Richard M. AMERICAN SIGN LANGUAGE AND DEAF CULTURE COMPETENCY OF OSTEOPATHIC MEDICAL STUDENTS. American Annals of the Deaf. Spr 2015;160(1):36-47. doi:10.1353/aad.2015.0014 
medRxiv preprint doi: https://doi.org/10.1101/2021.08.03.21261522; this version posted August 4, 2021. The copyright holder for this preprint (which was not certified by peer review) is the author/funder, who has granted medRxiv a license to display the preprint in perpetuity.

It is made available under a CC-BY-ND 4.0 International license .

42. Holzinger A, Lettner S, Franz A. Attitudes of dental students towards patients with special healthcare needs: Can they be improved? European Journal of Dental Education. May 2020;24(2):243-251. doi:10.1111/eje.12490

43. Saunder L, Knight RA. CitySCaPE: Moving beyond indifference in education for preregistration nurses about learning disability. Nurse education in practice. 2017;26:82-88. doi:http://dx.doi.org/10.1016/j.nepr.2017.07.008

44. Santoro JD, Whitgob EE, Huffman LC. Cluster Randomized Controlled Trial of Disability Education Module During Clinical Clerkship. Clinical Pediatrics. Nov 2019;58(13):1387-1393. doi:10.1177/0009922819850475

45. Perusini DJ, Llacuachaqui M, Sigal MJ, Dempster LJ. Dental Students' Clinical Expectations and Experiences Treating Persons with Disabilities. Journal of dental education. 2016;80(3):301-310.

46. Castro SS, Rowe M, Andrade LF, Cyrino EG. Developing competencies among health professions students related to the care of people with disabilities: a pilot study. InterfaceComunicacao Saude Educacao. Apr-Jun 2018;22(65):551-563. doi:10.1590/180757622016.0684

47. Burrola-Mendez Y, Goldberg M, Gartz R, Pearlman J. Development of a Hybrid Course on Wheelchair Service Provision for clinicians in international contexts. PLoS ONE. 2019;13(6):e0199251. doi:http://dx.doi.org/10.1371/journal.pone.0199251

48. Mohebbi SZ, Chinipardaz Z, Batebi A. Effectiveness of training senior dental students on oral health care for disabled patients. European Journal of Dental Education. Nov 2014;18(4):214-221. doi:10.1111/eje.12090

49. Ahmad MS, Mokhtar IW, Khan NLA. Extramural oral health educational program involving individuals with disabilities: Impact on dental students' professionalism. Journal of International Society of Preventive and Community Dentistry. May-Jun 2020;10(3):323-328. doi:10.4103/jispcd.JISPCD_74_20

50. Crane JM, Strickler JG, Lash AT, et al. Getting comfortable with disability: The shortand long-term effects of a clinical encounter. Disability and Health Journal. 2020:100993. doi:http://dx.doi.org/10.1016/j.dhjo.2020.100993

51. Tai S, Woodward-Kron R, Barr C. Audiology Students' Perspectives of Enacting and Learning Clinical Communication: A Qualitative Interview and Video Reflexivity Study. American Journal of Audiology. Jun 2018;27(2):219-230. doi:10.1044/2018_aja-17-0097

52. Ozkan F, Uslu N, Zincir H. Effect of practices for mentally disabled children on attitudes of nursing students. Cukurova Medical Journal. 2020;45(3):851-859. doi:10.17826/cumj.715000 53. Sinai A, Strydom A, Hassiotis A. Evaluation of medical students' attitudes towards people with intellectual disabilities: a naturalistic study in one medical school. Advances in Mental Health and Intellectual Disabilities. 2013 2020-09-14 2013;7(1):18-26. doi:http://dx.doi.org/10.1108/20441281311294666

54. Shields LBE, Peppas DS, Rosenberg E. Renal Calculus in Floating-Harbor Syndrome: A Case Report. Journal of Pediatric Health Care. Jan 2019;33(1):97-101. doi:10.1016/j.pedhc.2018.07.009

55. Watkins LV, Colgate R. Improving healthcare for people with intellectual disabilities: the development of an evidence-based teaching programme. Advances in Mental Health and Intellectual Disabilities. 2016;10(6):333-341. doi:10.1108/amhid-07-2016-0009

56. Forsgren E, Hartelius L, Saldert C. Improving medical students' knowledge and skill in communicating with people with acquired communication disorders. International journal of 
medRxiv preprint doi: https://doi.org/10.1101/2021.08.03.21261522; this version posted August 4, 2021. The copyright holder for this preprint (which was not certified by peer review) is the author/funder, who has granted medRxiv a license to display the preprint in perpetuity.

It is made available under a CC-BY-ND 4.0 International license .

speech-language pathology. 2017;19(6):541-550.

doi:http://dx.doi.org/10.1080/17549507.2016.1216602

57. McIntosh CE, Thomas CM, Wilczynski S, McIntosh DE. Increasing Nursing Students' Knowledge of Autism Spectrum Disorder by Using a Standardized Patient. Nursing education perspectives. 2018;39(1):32-34. doi:http://dx.doi.org/10.1097/01.NEP.0000000000000179

58. Woodard LJ, Havercamp SM, Zwygart KK, Perkins EA. An Innovative Clerkship Module Focused on Patients With Disabilities. Academic Medicine. Apr 2012;87(4):537-542. doi:10.1097/ACM.0b013e318248ed0a

59. Jones J, McQueen M, Lowe S, Minnes P, Rischke A. Interprofessional Education in Canada: Addressing Knowledge, Skills, and Attitudes Concerning Intellectual Disability for Future Healthcare Professionals. Journal of Policy and Practice in Intellectual Disabilities. Sep 2015;12(3):172-180. doi:10.1111/jppi.12112

60. Beverly BL, Wooster D. An Interprofessional Education Initiative for Allied Health Students Preparing to Serve Individuals with Autism Spectrum Disorders. Journal of allied health. 2018;47(2):90-95.

61. Coret A, Boyd K, Hobbs K, Zazulak J, McConnell M. Patient Narratives as a Teaching Tool: A Pilot Study of First-Year Medical Students and Patient Educators Affected by Intellectual/Developmental Disabilities. Teaching and Learning in Medicine. 2018;30(3):317327. doi:10.1080/10401334.2017.1398653

62. Jain S, Foster E, Biery N, Boyle V. Patients With Disabilities as Teachers. Family Medicine. Jan 2013;45(1):37-39.

63. Karl R, McGuigan D, Withiam-Leitch ML, Akl EA, Symons AB. Reflective Impressions of a Precepted Clinical Experience Caring for People With Disabilities. Intellectual and Developmental Disabilities. Aug 2013;51(4):237-245. doi:10.1352/1934-9556-51.4.237

64. Thomas B, Courtenay K, Hassiotis A, Strydom A, Rantell K. Standardised patients with intellectual disabilities in training tomorrow's doctors. BJPsych Bulletin. 2014;38(3):132-136. doi:http://dx.doi.org/10.1192/pb.bp.113.043547

65. Baylor C, Burns M, McDonough K, Mach H, Yorkston K. Teaching Medical Students Skills for Effective Communication With Patients Who Have Communication Disorders. American Journal of Speech-Language Pathology. Feb 2019;28(1):155-164. doi:10.1044/2018_ajslp-18-0130

66. Saunder L, Berridge EJ. Immersive simulated reality scenarios for enhancing students' experience of people with learning disabilities across all fields of nurse education. Nurse Education in Practice. Nov 2015;15(6):397-402. doi:10.1016/j.nepr.2015.04.007

67. Dyer K, Aubeeluck A, Yates NL, Das Nair R. A Multiple Timepoint Pre-post Evaluation of a 'Sexual Respect' DVD to Improve Competence in Discussing Sex with Patients with Disability. Sexuality and Disability. Sep 2015;33(3):385-397. doi:10.1007/s11195-014-9375-3 68. Provident IM, Colmer MA. Muscular dystrophy summer camp: A case study of a nontraditional level I fieldwork using a collaborative supervision model. Work. 2013;44(3):337-404. doi:10.3233/wor-121510

69. Adib-Hajbaghery M, Rezaei-Shahsavarloo Z. Nursing students' knowledge of and performance in communicating with patients with hearing impairment. Japan journal of nursing science : JJNS. 2015;12(2):135-44. doi:https://dx.doi.org/10.1111/jjns.12057

70. Tate JA, Newtz C, Ali A, Happ MB. Advancing Patient-Centered Communication Content for Prelicensure Nursing Students Using StudentSPEACS. Nurse Educator. Jul-Aug 2020;45(4):E36-E40. doi:10.1097/nne.0000000000000785 
medRxiv preprint doi: https://doi.org/10.1101/2021.08.03.21261522; this version posted August 4, 2021. The copyright holder for this preprint (which was not certified by peer review) is the author/funder, who has granted medRxiv a license to display the preprint in perpetuity. It is made available under a CC-BY-ND 4.0 International license .

71. Theoret C, Patel R, Thangamathesvaran L, Shah R, Chen S, Traba C. Creating DisabilityCompetent Medical Students Via Community Outreach. Journal of the National Medical Association. 2020; doi:http://dx.doi.org/10.1016/j.jnma.2020.07.010

72. Watters AL, Stabulas-Savage J, Toppin JD, Janal MN, Robbins MR. Incorporating Experiential Learning Techniques to Improve Self-Efficacy in Clinical Special Care Dentistry Education. Journal of Dental Education. Sep 2015;79(9):1016-1023.

73. Vento-Wilson MT, McGuire A, Ostergren JA. Role of the Speech-Language Pathologist Augmentative and Alternative Communication for Acute Care Patients With Severe Communication Impairments. Dimensions of Critical Care Nursing. Mar-Apr 2015;34(2):112119. doi:10.1097/dcc.0000000000000094

74. Kirshblum S, Murray R, Potpally N, Foye PM, Dyson-Hudson T, DallaPiazza M. An introductory educational session improves medical student knowledge and comfort levels in caring for patients with physical disabilities. Disability and Health Journal. Jan 2020;13(1)100825. doi:10.1016/j.dhjo.2019.100825

75. Read S, Rushton A. Cultivating understanding of health issues for adults with intellectual disability. Nurse Education Today. Sep 2013;33(9):1020-1025. doi:10.1016/j.nedt.2012.03.013

76. Major NE, Peacock G, Ruben W, Thomas J, Weitzman CC. Autism Training in Pediatric Residency: Evaluation of a Case-Based Curriculum. Journal of Autism and Developmental Disorders. May 2013;43(5):1171-1177. doi:10.1007/s10803-012-1662-1

77. Tuffrey-Wijne I, Rose T, Grant R, Wijne A. Communicating about death and dying: Developing training for staff working in services for people with intellectual disabilities. Journal of Applied Research in Intellectual Disabilities. Nov 2017;30(6):1099-1110. doi:10.1111/jar.12382

78. Toro ML, Bird E, Oyster M, et al. Development of a wheelchair maintenance training programme and questionnaire for clinicians and wheelchair users. Disability and rehabilitation Assistive technology. 2017;12(8):843-851. doi:http://dx.doi.org/10.1080/17483107.2016.1277792

79. Bogetz JF, Gabhart JM, Rassbach CE, et al. Outcomes of a Randomized Controlled Educational Intervention to Train Pediatric Residents on Caring for Children With Special Health Care Needs. Clinical Pediatrics. Jun 2015;54(7):659-666. doi:10.1177/0009922814564050

80. Casson I, Abells D, Boyd K, et al. Teaching family medicine residents about care of adults with intellectual and developmental disabilities. Canadian Family Physician. Apr 2019;65:S35-S40.

81. McGonigle JJ, Migyanka JM, Glor-Scheib SJ, et al. Development and evaluation of educational materials for pre-hospital and emergency department personnel on the care of patients with autism spectrum disorder. Journal of Autism and Developmental Disorders. 2014;44(5):1252-1259. doi:http://dx.doi.org/10.1007/s10803-013-1962-0

82. Doyle SD, Bennett S. Feasibility and effect of a professional education workshop for occupational therapists' management of upper-limb poststroke sensory impairment. The American journal of occupational therapy : official publication of the American Occupational Therapy Association. 2014;68(3):e74-e83. doi:http://dx.doi.org/10.5014/ajot.2014.009019

83. Dunleavy K, Chevan J, Sander AP, Gasherebuka JD, Mann M. Application of a contextual instructional framework in a continuing professional development training program for physiotherapists in Rwanda. Disability and Rehabilitation. 2018;40(13):1600-1608. doi:10.1080/09638288.2017.1300692 
medRxiv preprint doi: https://doi.org/10.1101/2021.08.03.21261522; this version posted August 4, 2021. The copyright holder for this preprint (which was not certified by peer review) is the author/funder, who has granted medRxiv a license to display the preprint in perpetuity. It is made available under a CC-BY-ND 4.0 International license .

84. Mazurek MO, Parker RA, Chan J, Kuhlthau K, Sohl K, Collaborative EA. Effectiveness of the Extension for Community Health Outcomes Model as Applied to Primary Care for Autism A Partial Stepped-Wedge Randomized Clinical Trial. Jama Pediatrics. May 2020;174(5)e196306. doi:10.1001/jamapediatrics.2019.6306

85. Locke HN, Doctors S, Randriamampianina I, Chamberlain MA, O'Connor RJ. EVALUATING A GLOBAL HEALTH PARTNERSHIP REHABILITATION TRAINING PROGRAMME IN MADAGASCAR. Journal of Rehabilitation Medicine. Dec 2019;51(11):847-853. doi:10.2340/16501977-2621

86. Edinger ZS, Powers KA, Jordan KS, Callaway DW. Evaluation of an online educational intervention to increase knowledge and self-efficacy in disaster responders and critical care transporters caring for individuals with developmental disabilities. Disaster Medicine and Public Health Preparedness. 2019;13(4):677-681.

87. Auberry K, Wills K, Shaver C. Improving medication practices for persons with intellectual and developmental disability: Educating direct support staff using simulation, debriefing, and reflection. Journal of Intellectual Disabilities. 2019;23(4):498-511. doi:http://dx.doi.org/10.1177/1744629517731231

88. Olowoyeye AO, Musa KO, Aribaba OT. Outcome of training of maternal and child health workers in Ifo Local Government Area, Ogun State, Nigeria, on common childhood blinding diseases: a pre-test, post-test, one-group quasi-experimental study. Bmc Health Services Research. Jun 2019;19430. doi:10.1186/s12913-019-4272-1

89. Cameron A, McPhail SM, Hudson K, Fleming J, Lethlean J, Finch E. A pre-post intervention study investigating the confidence and knowledge of health professionals communicating with people with aphasia in a metropolitan hospital. Aphasiology. 2017;31(3):359-374. doi:http://dx.doi.org/10.1080/02687038.2016.1225277

90. Catteau C, Faulks D, Mishellany-Dutour A, et al. Using e-learning to train dentists in the development of standardised oral health promotion interventions for persons with disability. European Journal of Dental Education. Aug 2013;17(3):143-153. doi:10.1111/eje.12024 91. Quinn BL, Smolinski M. Improving School Nurse Pain Assessment Practices for Students With Intellectual Disability. Journal of School Nursing. Dec 2018;34(6):480-488. doi:10.1177/1059840517722591

92. Phlypo I, De Tobel J, Marks L, De Visschere L, Koole S. Integrating community service learning in undergraduate dental education: A controlled trial in a residential facility for people with intellectual disabilities. Special Care in Dentistry. Jul-Aug 2018;38(4):201-207. doi:10.1111/scd.12298

93. Sanchez D, Adamovich S, Ingram M, et al. The potential in preparing community health workers to address hearing loss. Journal of the American Academy of Audiology. 2017;28(6):562-574. doi:http://dx.doi.org/10.3766/jaaa.16045

94. Figueiras ACM, Puccini RF, Silva EMK. Continuing education on child development for primary healthcare professionals: a prospective before-and-after study. Sao Paulo Medical Journal. 2014;132(4):211-218. doi:10.1590/1516-3180.2014.1324665 